\title{
Varieties of Regular Pseudocomplemented de Morgan Algebras
}

\author{
M. E. Adams, H. P. Sankappanavar, and Júlia Vaz de Carvalho*
}

This paper has appeared in the journal Order (published online on January 10, 2020).

\begin{abstract}
In this paper, we investigate the varieties $\mathbf{M}_{n}$ and $\mathbf{K}_{n}$ of regular pseudocomplemented de Morgan and Kleene algebras of range $n$, respectively. Priestley duality as it applies to pseudocomplemented de Morgan algebras is used. We characterise the dual spaces of the simple (equivalently, subdirectly irreducible) algebras in $\mathbf{M}_{n}$ and explicitly describe the dual spaces of the simple algebras in $\mathbf{M}_{1}$ and $\mathbf{K}_{1}$. We show that the variety $\mathbf{M}_{1}$ is locally finite, but this property does not extend to $\mathbf{M}_{n}$ or even $\mathbf{K}_{n}$ for $n \geq 2$. We also show that the lattice of subvarieties of $\mathbf{K}_{1}$ is an $\omega+1$ chain and the cardinality of the lattice of subvarieties of either $\mathbf{K}_{2}$ or $\mathbf{M}_{1}$ is $2^{\omega}$. A description of the lattice of subvarieties of $\mathbf{M}_{1}$ is given.
\end{abstract}

2010 Mathematics subject classification: Primary 06D30, 06D15, 03G25; Secondary 08B15, 06D50, $03 G 10$. Keywords: variety of algebras, lattice of subvarieties, regular pseudocomplented de Morgan algebra (of range $n$ ), discriminator variety, simple algebra, subdirectly irreducible algebra, Priestley duality.

\section{Introduction}

A pseudocomplemented de Morgan algebra ( $p m$-algebra for short) is an algebra $\left(L ; \wedge, \vee,{ }^{*},{ }^{\prime}, 0,1\right)$ of signature $(2,2,1,1,0,0)$ such that $\left(L ; \wedge, \vee,{ }^{*}, 0,1\right)$ is a pseudocomplemented distributive algebra and $\left(L ; \wedge, \vee,^{\prime}, 0,1\right)$ is a de Morgan algebra, that is $(L ; \wedge, \vee, 0,1)$ is a bounded distributive lattice, ${ }^{*}$ satisfies, for $x, y \in L, x \wedge y=0$ if and only if $y \leq x^{*}$, whilst ' satisfies $(x \vee y)^{\prime}=x^{\prime} \wedge y^{\prime}$, $(x \wedge y)^{\prime}=x^{\prime} \vee y^{\prime}, 0^{\prime}=1,1^{\prime}=0$, and $x^{\prime \prime}=x$.

Since, as shown by Ribenboim [19, the class of pseudocomplemented distributive algebras form a variety, so too does the class of pseudocomplemented de Morgan algebras.

Pseudocomplemented de Morgan algebras were first investigated in Romanowska [20] and have been considered by several authors since (see, for example, Denecke [7, Gaitán [8], Guzmán and Squier [9], as well as [21, 22], 23], 24], 28]). Romanowska's characterisation of finite subdirectly irreducible $p m$-algebras in 20, was extended to a characterisation of all subdirectly irreducible pm-algebras in 21] (for those algebras which are non-regular) and in 23] (for those algebras which are regular). An algebra is regular if any two congruences on it are equal whenever they have a class in common. It was in 21] that regularity in $p m$-algebras was first considered. It is better understood in the context of regular double $p$-algebras where they have been studied extensively (see, for example, Beazer [3], Katriňák [13, Koubek and Sichler [12, Taylor [25], Varlet [27, as

*This work was partially supported by the Fundação para a Ciência e Tecnologia (Portuguese Foundation for Science and Technology) through the projects UID/MAT/00297/2013 and UID/MAT/00297/2019 (Centro de Matemática e Aplicações). 
well as [1).

As observed by Romanowska, for any element $a$ in a $p m$-algebra, $a^{+}=a^{\prime * \prime}$ is its dual pseudocomplement. That is, every $p m$-algebra $L=\left(L ; \wedge, \vee,{ }^{*},{ }^{\prime}, 0,1\right)$ gives rise to a distributive double $p$-algebra $L_{d p}=\left(L ; \wedge, \vee,{ }^{*},{ }^{+}, 0,1\right)$. The determination congruence $\Phi$ on a double $p$-algebra $\left(L ; \wedge, \vee,{ }^{*},{ }^{+}, 0,1\right)$ is the congruence given by, for $x, y \in L$

$$
x \equiv y(\Phi) \text { iff } x^{*}=y^{*} \text { and } x^{+}=y^{+} .
$$

As shown by Varlet [27, a double $p$-algebra is regular if and only if $\Phi=\triangle$, the equality congruence. The Moisil congruence $\Psi$ on a $p m$-algebra $\left(L ; \wedge, \vee,{ }^{*},{ }^{\prime}, 0,1\right)$ is the congruence given by, for $x, y \in L$,

$$
x \equiv y(\Psi) \text { iff } x^{*}=y^{*} \text { and } x^{*}=y^{\prime *}
$$

As shown in 21, a $p m$-algebra $L$ is regular if and only if $\Psi=\triangle$. Noticing that $x^{\prime *}=y^{\prime *}$ is equivalent to $x^{\prime * \prime}=y^{\prime * \prime}$, we have that $L$ is regular if and only if the distributive double $p$-algebra $L_{d p}$ is regular. That the class of regular double $p$-algebras is a variety, namely the subvariety of distributive double $p$-algebras determined by the inequality

$$
x \wedge x^{+} \leq y \vee y^{*},
$$

was shown by Katriňák [13. It follows immediately that the class of regular pm-algebras is a variety, namely the subvariety of $p m$-algebras determined by the inequality

$$
x \wedge x^{* \prime} \leq y \vee y^{*} .
$$

Let $\left(L ; \wedge, \vee,^{*}{ }^{\prime}, 0,1\right)$ be a $p m$-algebra. For $x \in L$ and $n<\omega$, define $x^{n(\prime *)}$ recursively as follows:

$$
x^{0(*)}=x \text { and } x^{(n+1)(/ *)}=\left(x^{n(*)}\right)^{\prime *} .
$$

For any element $x$ of a distributive double $p$-algebra, $x \geq x^{+*}$, that is, for any $x \in L, x \geq x^{\prime * * *}$. Further, since for $x, y \in L,(x \vee y)^{*}=x^{*} \wedge y^{*}$, it follows that $x \wedge x^{\prime *} \geq x^{\prime *} \wedge x^{\prime * \prime *}=\left(x^{\prime} \vee x^{\prime * \prime}\right)^{*}=$ $\left(x \wedge x^{\prime *}\right)^{\prime *}$. In other words, it is always the case that

$$
x \wedge x^{\prime *} \geq\left(x \wedge x^{\prime *}\right)^{\prime *} \geq\left(x \wedge x^{\prime *}\right)^{2(*)} \geq \ldots
$$

As such, $L$ is said to have finite range providing, for each $x \in L$, there exists $n<\omega$ such that $\left(x \wedge x^{\prime *}\right)^{n(\prime *)}=\left(x \wedge x^{\prime *}\right)^{(n+1)(/ *)}$. Further, if, for some $n<\omega,\left(x \wedge x^{\prime *}\right)^{n(1 *)}=\left(x \wedge x^{\prime *}\right)^{(n+1)(1 *)}$ for every $x \in L$, then $L$ is said to be of range $n$. Obviously, if $L$ is of range $n$, it is also of range $m$, for any $m \geq n$.

A variety of (regular) pm-algebras is said to be of range $n$ if all its members are of range $n$. For a variety $\mathbf{V}, L_{V}(\mathbf{V})$ denotes the lattice of all subvarieties of $\mathbf{V}$.

Although it appears to be somewhat ad hoc, the notion of finite range occurs naturally in the study of $p m$-algebras. For example, in 21] it is shown that for $L$ to be regular with finite range is equivalent to the compact elements forming a Boolean sublattice of the congruence lattice of $L$. Also from [21, for each $n<\omega$, the variety of regular $p m$-algebras of range $n$ is a discriminator variety and so, in particular, it has equationally definable principal congruences, every subdirectly irreducible algebra is simple, and any subalgebra of a simple algebra is simple. In 22, it is shown that $L$ has finite range if and only if every principal congruence on $L$ is a join of finitely many principal lattice congruences on $L$.

Further, in 22, the variety $\mathbf{P M}_{0}$ (there denoted by $V_{0}$ ) of the $p m$-algebras of range 0 is considered and its subdirectly irreducible algebras are described in order to determine the lattice 
$L_{V}\left(\mathbf{P M}_{0}\right)$ of its subvarieties. We remark that $L_{V}\left(\mathbf{P M}_{0}\right)$ is isomorphic to the 11-element lattice $\mathbf{1} \oplus(\mathbf{2} \times \mathbf{5})$ (a correction to the lattice presented in [22, Corollary 6.7], since the subvarieties there denoted by $V\left(L_{3}\right)$ and $V\left(D_{4}\right)$ are actually incomparable). Having determined $L_{V}\left(\mathbf{P M}_{0}\right)$, it was natural to ask for a description of $L_{V}\left(\mathbf{P M}_{1}\right), L_{V}\left(\mathbf{P M}_{2}\right), \ldots$ where $\mathbf{P} \mathbf{M}_{n}$ denotes the variety of $p m$-algebras of range $n$. That question, raised in [22, was the primary motivation behind this paper.

Let $\mathbf{M}$ denote the variety of regular pseudocomplemented de Morgan algebras and, for $n<\omega$, let $\mathbf{M}_{n} \subseteq \mathbf{P M}_{n}$ denote the subvariety of $\mathbf{M}$ that satisfies $\left(x \wedge x^{\prime *}\right)^{n(\prime *)} \approx\left(x \wedge x^{\prime *}\right)^{(n+1)(\prime *)}$, that is, $\mathbf{M}_{n}=\mathbf{M} \cap \mathbf{P} \mathbf{M}_{n}$ is the variety of regular pseudocomplemented de Morgan algebras of range $n$. In this paper, it will be shown that, even for the better behaved subvariety $\mathbf{M}_{n}$ of $\mathbf{P} \mathbf{M}_{n}, L_{V}\left(\mathbf{M}_{n}\right)$ is already quite complex.

As shown by Kalman [11, the lattice of subvarieties of the variety of de Morgan algebras is a 4 -element chain whose members are, from the least to the greatest, the trivial variety, the variety of Boolean algebras, the variety of Kleene algebras, and the variety of de Morgan algebras. As determined by Kalman, a Kleene algebra is a de Morgan algebra that satisfies the inequality

$$
x \wedge x^{\prime} \leq y \vee y^{\prime} .
$$

In the present context, let $\mathbf{K}$ denote the variety of regular pseudocomplemented Kleene algebras and, for $n<\omega$, let $\mathbf{K}_{n}$ denote the subvariety of $\mathbf{K}$ that satisfies $\left(x \wedge x^{\prime *}\right)^{n(\prime *)} \approx\left(x \wedge x^{\prime *}\right)^{(n+1)(\prime *)}$, that is, $\mathbf{K}_{n}=\mathbf{K} \cap \mathbf{P} \mathbf{M}_{n}$ is the variety of regular pseudocomplemented Kleene algebras of range $n$. Obviously, $\mathbf{K}_{n} \subseteq \mathbf{M}_{n} \subseteq \mathbf{P M}_{n}$. Typically there is a significant difference between Kleene algebras and de Morgan algebras and, although this is reflected initially in a sharp difference between $L_{V}\left(\mathbf{K}_{0}\right)$ and $L_{V}\left(\mathbf{M}_{0}\right)$, as well as between $L_{V}\left(\mathbf{K}_{1}\right)$ and $L_{V}\left(\mathbf{M}_{1}\right)$, thereafter this difference is short lived.

A simple inspection of the description of the subdirectly irreducible algebras in the variety $\mathbf{P M}_{0}$ presented in [22, Theorem 6.5] leads to the conclusion that $L_{V}\left(\mathbf{M}_{0}\right)$ is isomorphic to the 5-element lattice $\mathbf{1} \oplus(\mathbf{2} \times \mathbf{2})$ and that $L_{V}\left(\mathbf{K}_{0}\right)$ is isomorphic to a 3 -element chain. In Sections $\mathbf{5}$ and 6 we will show that $L_{V}\left(\mathbf{K}_{1}\right)$ is isomorphic to an $\omega+1$ chain (Theorem [5.4), $\left|L_{V}\left(\mathbf{M}_{1}\right)\right|=2^{\omega}$ (Corollary 5.7) and $\left|L_{V}\left(\mathbf{K}_{2}\right)\right|=2^{\omega}$ (Theorem 6.5). Summarising we have the following theorems.

THEOREM 1.1. For the variety $\mathbf{K}$, we have

(1) $L_{V}\left(\mathbf{K}_{0}\right)$ is isomorphic to a 3-element chain,

(2) $L_{V}\left(\mathbf{K}_{1}\right)$ is isomorphic to an $\omega+1$ chain,

(3) $\left|L_{V}\left(\mathbf{K}_{2}\right)\right|=2^{\omega}$.

THEOREM 1.2. For the variety $\mathbf{M}$, we have

(1) $L_{V}\left(\mathbf{M}_{0}\right)$ is isomorphic to the 5-element lattice $\mathbf{1} \oplus(\mathbf{2} \times \mathbf{2})$,

(2) $\left|L_{V}\left(\mathbf{M}_{1}\right)\right|=2^{\omega}$.

Local finiteness in the varieties $\mathbf{M}_{n}$ and $\mathbf{K}_{n}, 1 \leq n<\omega$, is also analysed in Theorems 4.10 and 4.13 allowing us to establish the following result.

THEOREM 1.3. For the varieties $\mathbf{K}$ and $\mathbf{M}$,

(1) $\mathbf{M}_{1}$ is locally finite (de facto, so too is $\mathbf{K}_{1}$ ),

(2) $\mathbf{K}_{2}$ is not locally finite, in fact the free algebra on one generator $F_{\mathbf{K}_{2}}(1)$ is infinite (de facto, for $n \geq 2, \mathbf{K}_{n}$ and $\mathbf{M}_{n}$ are not locally finite and the respective free algebras on one generator are infinite). 
Even though $L_{V}\left(\mathbf{M}_{1}\right)$ is uncountable, local finiteness of $\mathbf{M}_{1}$ allows us to have a genuine understanding of the structure of $L_{V}\left(\mathbf{M}_{1}\right)$, the key to which is given in Theorem [5.6. The discussion following Corollary 5.7 contains the details. At the cost of some transparency, that discussion is summarised in Theorem 5.8

To establish the aforementioned results, Priestley duality as it applies to pseudocomplemented de Morgan algebras is used extensively and a brief outline of this duality is given in Section 2 , In Section 3 we characterise, via their dual spaces, the regular $p m$-algebras of range $n$. It will be seen that the apparently ad hoc nature of finite range is not ad hoc at all and, in fact, is a natural property of Priestley duality, namely the notion of $\zeta$-distance, as seen in Section 3 A characterisation, via dual spaces, of the simple (equivalently, subdirectly irreducible) algebras in $\mathbf{M}_{n}$ and a description of the dual spaces of the simple algebras in $\mathbf{M}_{1}$ and $\mathbf{K}_{1}$ are given in Section 4. It is also in this section that we prove Theorem 1.3. The contents of Sections 5 and 6 have already been described above. Finally, in Section ?? we present some remarks on connections between regular $p m$-algebras and de Morgan Heyting algebras.

\section{Preliminaries}

We refer to [4] and to [2] for basic concepts and results on universal algebra and on distributive $p$-algebras, respectively. We point out that, for us, subdirectly irreducible algebras and simple algebras are non-trivial.

We will use Priestley duality as it applies to pseudocomplemented de Morgan algebras. Here we give a brief outline.

Let $(P ; \leq)$ be a partially ordered set. We denote by $\operatorname{Min}(P)$ and $\operatorname{Max}(P)$ the sets of minimal elements of $P$ and of maximal elements of $P$, respectively. For any $X \subseteq P$, let $(X]=\{y \in$ $P: y \leq x$ for some $x \in X\}$, and $[X)=\{y \in P: y \geq x$ for some $x \in X\}$. The subset $X$ is said to be decreasing if $(X]=X$, and increasing if $[X)=X$. Define also $\operatorname{Min}(X)=\operatorname{Min}(P) \cap(X]$ and $\operatorname{Max}(X)=\operatorname{Max}(P) \cap[X)$. Should $X=\{x\}$, the sets $(X],[X), \operatorname{Min}(X)$ and $\operatorname{Max}(X)$ will be denoted by $(x],[x), \operatorname{Min}(x)$ and $\operatorname{Max}(x)$, respectively.

An ordered topological space $P=(P ; \tau, \leq)$ is a Priestley space if it is a compact totally orderdisconnected space, that is $(P ; \tau)$ is a compact topological space, $(P ; \leq)$ is a partially ordered set, and, for $x, y \in P$, if $y \not \leq x$, then there exists a clopen decreasing set $X$ such that $x \in X$ and $y \notin X$. In [16, Priestley showed that the category of all Priestley spaces together with all continuous order-preserving maps (that is, for a continuous map $\varphi: P \rightarrow Q$, if $x \leq y$, then $\varphi(x) \leq \varphi(y)$ ) is dually equivalent to the category of all bounded distributive lattices together with all $\{0,1\}$-lattice homomorphisms (see also [18]).

Priestley [17] extended her duality to the category of distributive pseudocomplemented lattices by showing that the category of distributive pseudocomplemented lattices together with all lattice homomorphisms that preserve * is dually equivalent to the category of all $p$-spaces together with all p-morphisms. A $p$-space is a Priestley space $P=(P ; \tau, \leq)$ such that, whenever $X \subseteq P$ is a clopen decreasing set, then $[X]$ is clopen. Given $p$-spaces $P$ and $Q$, a map $\varphi: P \rightarrow Q$ is a $p$-morphism if it is continuous order-preserving and, for any $x \in P, \operatorname{Min}(\varphi(x)) \subseteq \varphi(\operatorname{Min}(x))$, which implies that $\operatorname{Min}(\varphi(x))=\varphi(\operatorname{Min}(x))$. 
Meanwhile, Cornish and Fowler [5] extended Priestley's duality to the category of de Morgan algebras by showing that the category of de Morgan algebras together with all lattice homomorphisms that preserve' ${ }^{\prime}$ is dually equivalent to the category of all $m$-spaces together with all $m$-morphisms. An $m$-space is a quadruple $P=(P ; \tau, \leq, \zeta)$ such that $(P ; \tau, \leq)$ is a Priestley space and $\zeta$ is a continuous order-reversing map from $P$ to $P$ satisfying $\zeta^{2}(x)=x$ for every $x \in P$. Given $m$-spaces $P$ and $Q$, a map $\varphi: P \rightarrow Q$ is an $m$-morphism if it is continuous order-preserving and $\varphi \circ \zeta=\zeta \circ \varphi$.

To summarise: $P=(P ; \tau, \leq, \zeta)$ is a pm-space if $(P ; \tau, \leq)$ is a Priestley space, $[X)$ is clopen for every clopen decreasing set $X \subseteq P, \zeta$ is a continuous order-reversing involution on $P$; for $p m$-spaces $P$ and $Q, \varphi: P \rightarrow Q$ is a pm-morphism if $\varphi$ is continuous, order-preserving, $\operatorname{Min}(\varphi(x)) \subseteq \varphi(\operatorname{Min}(x))$ for every $x \in P$, and $\varphi \circ \zeta=\zeta \circ \varphi$. The category of all pseudocomplemented de Morgan algebras together with all homomorphisms is dually equivalent to the category of all $p m$-spaces together with all $p m$-morphisms. In particular, for a $p m$-algebra $\left(L ; \wedge, \vee,{ }^{*},{ }^{\prime}, 0,1\right)$, its dual space is the pm-space $D(L)=(P ; \tau, \leq, \zeta)$ where $P$ is the set of prime ideals of $L$ ordered by inclusion, the topology $\tau$ has sub-basis $\left\{X_{a}: a \in L\right\} \cup\left\{P \backslash X_{a}: a \in L\right\}$ where $X_{a}=\{I \in P: a \notin I\}$ and $\zeta$ is defined by $\zeta(I)=\left\{a \in L: a^{\prime} \notin I\right\}$ for every $I \in P$. For a pm-space $(P ; \tau, \leq, \zeta)$, its dual pmalgebra is the algebra $E(P)=\left(L ; \cap, \cup,{ }^{*},{ }^{\prime}, \emptyset, P\right)$ where $L$ consists of all clopen decreasing subsets of $P$ and, for a clopen decreasing $X \subseteq P, X^{*}=P \backslash[X)$ and $X^{\prime}=P \backslash \zeta^{-1}(X)=P \backslash \zeta(X)=\zeta(P \backslash X)$.

For bounded distributive lattices $L$ and $K$ whose dual Priestley spaces are $P$ and $Q$, respectively, each homomorphism $f: L \rightarrow K$ is associated with a continuous order-preserving map $\varphi: Q \rightarrow P$ which is defined by $\varphi(J)=f^{-1}(J)$, for any prime ideal $J$ of $K$. Under Priestley duality, $f$ is one-to-one if and only if $\varphi$ is onto and $f$ is onto if and only if $\varphi$ is an order-embedding. From the latter, it follows that homomorphic images of $L$ correspond to closed subspaces of $P$ (ordered by the restriction of the order in $P$ ). For $p m$-spaces, we know that the $p m$-morphisms are continuous order-preserving maps $\varphi: Q \rightarrow P$ such that, for every $x \in Q, \operatorname{Min}(\varphi(x)) \subseteq \varphi(\operatorname{Min}(x))$ and $\varphi \circ \zeta=\zeta \circ \varphi$. Hence, it follows that the closed subspaces of $P$, for which $\operatorname{Min}(x)$ is a subset whenever $x$ is a member and are also closed under $\zeta$, correspond to the homomorphic images of $L$. We refer to all such subspaces as pm-subspaces of $P$.

As observed by Cornish and Fowler [6], an $m$-space $(P ; \tau, \leq, \zeta)$ corresponds to a Kleene algebra precisely when either $x \leq \zeta(x)$ or $\zeta(x) \leq x$ for every $x \in P$. In particular, the category of all pseudocomplemented Kleene algebras together with all homomorphisms is dually equivalent to the category of all $p m$-spaces $(P ; \tau, \leq, \zeta)$ such that either $x \leq \zeta(x)$ or $\zeta(x) \leq x$ for every $x \in P$, together with all $p m$-morphisms.

If a pm-morphism is a homeomorphism and an order-isomorphism, we say that it is a pmisomorphism and that the pm-spaces involved are pm-isomorphic.

Notice that if $(P ; \tau, \leq, \zeta)$ is a $p m$-space then $\zeta$ is a homeomorphism and a dual order-isomorphism from $P$ onto $P$.

It is well known that finite subsets of a Hausdorff space are closed and, consequently, an element $x$ is an isolated point if and only if $\{x\}$ is clopen. Recall also that a compact Hausdorff space is finite if and only if the topology is the discrete one. Stone spaces (that is, compact totally disconnected topological spaces) and Priestley spaces are, in particular, compact and Hausdorff.

Given a closed subset $X$ of a Priestley space, $[X)$ and $(X]$ are closed and if $X$ is decreasing 
and $y \notin X$ there exists a clopen increasing set $Y$ such that $y \in Y$ and $Y \cap X=\emptyset$.

If $(P ; \tau, \leq, \zeta)$ is the $p m$-space of a pseudocomplemented de Morgan algebra $L$, then $(P ; \tau, \leq)$ is the Priestley space of the distributive double $p$-algebra $L_{d p}$. Consequently, $\operatorname{Min}(P)$ and $\operatorname{Max}(P)$ are closed, since the set of minimal elements in the Priestley space of any distributive pseudocomplemented lattice is closed [17] and dually the set of maximal elements in the Priestley space of any double $p$-algebra is also closed.

Combining an observation of Cornish and Fowler [6] with a result due to Adams (cf. [17), we know that given a pseudocomplemented de Morgan algebra $L$ whose $p m$-space is $P=(P ; \tau, \leq, \zeta)$, there is a lattice-isomorphism from the lattice of congruences of $L$ onto the lattice of open sets $X$ of $P$ that satisfy $[\operatorname{Min}(P) \cap X) \subseteq X$ and $\zeta(X) \subseteq X$ (thus $\zeta(X)=X$ ). These open sets also satisfy $(\operatorname{Max}(P) \cap X] \subseteq X$, since $(\operatorname{Max}(P) \cap X]=\zeta([\operatorname{Min}(P) \cap X)) \subseteq \zeta(X)=X($ that $(\operatorname{Max}(P) \cap X] \subseteq X$ also follows from the fact that any congruence on $L$ is also a congruence on $L_{d p}$ ).

\section{$3 \quad$ Regularity and finite range}

In this section, we characterise, via their dual spaces, the regular pseudocomplemented de Morgan algebras and which amongst these algebras have finite range $n$. Although at first flush the notion of finite range may have seemed a little artificial, as Corollary 3.7] shows, in the context of pseudocomplemented de Morgan algebras, it really does arise naturally.

THEOREM 3.1. Let $L=\left(L ; \wedge, \vee,{ }^{*},{ }^{\prime}, 0,1\right)$ be a pm-algebra and $(P ; \tau, \leq, \zeta)$ its dual pm-space. We have that $L$ is regular if and only if $(P ; \leq)$ has height at most 1 , that is, all chains in $(P ; \leq)$ have at most 2 elements.

Proof. Recall that $L$ is regular if and only if its associated distributive double $p$-algebra $L_{d p}$ is regular, which, by [26, Theorem 3], is equivalent to every chain of prime ideals of $L$ having at most two elements, that is $(P ; \leq)$ having height at most 1 .

In the context of the previous result, we have that $L$ is regular if and only if $P=\operatorname{Min}(P) \cup$ $\operatorname{Max}(P)$.

In what follows, we consider that $n<\infty$, for all $n<\omega$.

Let $(P ; \leq)$ be a poset. The comparability graph of $P$ is the undirected graph with $P$ as the set of vertices and in which there is an edge between $x$ and $y$ if and only if $x \neq y$ and $x$ and $y$ are comparable in the poset. For $x, y \in P$, the distance between $x$ and $y$, denoted $\ell(x, y)$, is 0 if $x=y$ and otherwise is the length of a shortest (finite) path between $x$ and $y$ in the comparability graph of $P$, if such a path exists. In this case we say that the distance between $x$ and $y$ is finite, otherwise the distance is said to be infinite and we write $\ell(x, y)=\infty$.

An order component $Q \subseteq P$ is a non-empty set such that, for any $x, y \in Q, \ell(x, y)$ is finite and, for any $x \in Q$ and any $y \notin Q, \ell(x, y)$ is infinite. The order components of $(P ; \leq)$ form a partition of $P$ and we denote by $Q_{x}$ the order component to which $x \in P$ belongs.

For $X \subseteq P$ and $x \in P$, the distance of $x$ from $X$, denoted $\ell(x, X)$, is the least $\ell(x, y)$ with $y \in X$ if $X \neq \emptyset$, and $\ell(x, \emptyset)=\infty$. 
Let $P$ be a $p m$-space. Since $\zeta$ is an involutive dual order-isomorpism on $P$, it is obvious that, for any $x, y \in P$ and $X \subseteq P$, we have $\ell(x, y)=\ell(\zeta(x), \zeta(y)), \ell(x, \zeta(y))=\ell(\zeta(x), y)$ and $\ell(x, X)=\ell(\zeta(x), \zeta(X))$. Moreover, if $Q$ is an order component of $P$, then $\zeta(Q)$ is also an order component and, consequently, either $Q \cap \zeta(Q)=\emptyset$ or $Q=\zeta(Q)$.

LEMMA 3.2. Let $P$ be a pm-space of height at most 1 and $X \subseteq P$ be a clopen decreasing set. Then we have:

For $n<\omega$,

$$
X^{n(*)}=\{x: \ell(x,(P \backslash X))>n\} \text {, if } n \text { is even, }
$$

and,

$$
X^{n(*)}=\{x: \ell(x, \zeta(P \backslash X))>n\} \text {, if } n \text { is odd. }
$$

Moreover, $\ell(x,(P \backslash X))$ is infinite precisely when $Q_{x} \subseteq X$ and $\ell(x, \zeta(P \backslash X))$ is infinite precisely when $\zeta\left(Q_{x}\right) \subseteq X$ and

Proof. Let $X \subseteq P$ be a clopen decreasing set.

We begin by proving the last statement. Let $x \in P$. It is obvious that $\ell(x,(P \backslash X))$ is infinite if and only if $Q_{x} \cap(P \backslash X)=\emptyset$ or, equivalently, $Q_{x} \subseteq X$, and that $\ell(x, \zeta(P \backslash X))$ is infinite precisely when $Q_{x} \cap \zeta(P \backslash X)=\emptyset$, that is, since $\zeta$ is an involution, $\zeta\left(Q_{x}\right) \cap(P \backslash X)=\emptyset$ or, equivalently, $\zeta\left(Q_{x}\right) \subseteq X$.

Now we prove, inductively, the first part. As $X^{0(*)}=X$, it is obvious that $X^{0(/ *)}=$ $\{x: \ell(x,(P \backslash X))>0\}$.

Proceed inductively. For $n$ even, suppose that $X^{n(\prime *)}=\{x: \ell(x,(P \backslash X))>n\}$. Hence, we have $P \backslash X^{n(*)}=\{x: \ell(x,(P \backslash X)) \leq n\}$. Since $\zeta$ is a dual order-isomorphism, we get $X^{n(* *) \prime}=$ $\zeta\left(P \backslash X^{n(*)}\right)=\{\zeta(x): \ell(x,(P \backslash X)) \leq n\}=\{\zeta(x): \ell(\zeta(x), \zeta(P \backslash X)) \leq n\}=\{x: \ell(x, \zeta(P \backslash X)) \leq n\}$. Thus,

$$
X^{n(\prime *) \prime}=\{x: \ell(x, \zeta(P \backslash X)) \leq n\} .
$$

By definition, $X^{(n+1)(/ *)}=P \backslash\left[X^{n(\prime *)}\right)$. Obviously, $\{x: \ell(x, \zeta(P \backslash X))>n+1\} \subseteq X^{(n+1)(/ *)}$, in view of (11). Let $x \in X^{(n+1)(*)}$. So, we have, by (11), that $\ell(x, \zeta(P \backslash X)) \not \leq n$. We also claim that $\ell(x, \zeta(P \backslash X))>n+1$. For, suppose $\ell(x, \zeta(P \backslash X))=n+1$. Then $\ell(x, z)=n+1$, for some $z \in \zeta(P \backslash X)$. As $n+1$ is odd, we have that $x \in \operatorname{Max}(P)$ and $z \in \operatorname{Min}(P)$, or $x \in \operatorname{Min}(P)$ and $z \in \operatorname{Max}(P)$. The latter yields a contradiction, since, as $\zeta(P \backslash X)$ is decreasing, there would exist $z_{1} \in \zeta(P \backslash X)$ such that $\ell\left(x, z_{1}\right)=n$ and so $\ell(x, \zeta(P \backslash X)) \leq n$. Thus $x \in \operatorname{Max}(P)$ and $z \in \operatorname{Min}(P)$ and then $x>y$, for some $y$ such that $\ell(y, \zeta(P \backslash X))=n$. So $x \in\left[X^{n(\prime *) \prime}\right)$, a contradiction, proving the claim. Thus $X^{(n+1)(*)} \subseteq\{x: \ell(x, \zeta(P \backslash X))>n+1\}$ and we conclude that $X^{(n+1)(*)}=\{x: \ell(x, \zeta(P \backslash X))>n+1\}$.

Now, as $X^{(n+1)(*)}=\{x: \ell(x, \zeta(P \backslash X))>n+1\}$ and $\zeta$ is an involutive dual order-isomorphism, we have $X^{(n+1)(\prime *) \prime}=\zeta\left(P \backslash X^{(n+1)(\prime *)}\right)=\{\zeta(x): \ell(x, \zeta(P \backslash X)) \leq n+1\}=\{\zeta(x): \ell(\zeta(x),(P \backslash X)) \leq$ $n+1\}=\{x: \ell(x,(P \backslash X)) \leq n+1\}$. By definition, $X^{(n+2)(1 *)}=P \backslash\left[X^{(n+1)(\prime *) \prime}\right)$. It is obvious that $\{x: \ell(x,(P \backslash X))>n+2\} \subseteq X^{(n+2)(*)}$. Let $x \in X^{(n+2)(* *)}$. Then $\ell(x,(P \backslash X))>n+1$. Suppose $\ell(x,(P \backslash X))=n+2$. Then $\ell(x, z)=n+2$, for some $z \in P \backslash X$. As $n+2$ is even, we have that $x, z \in \operatorname{Max}(P)$ or $x, z \in \operatorname{Min}(P)$. The latter yields a contradiction, since, as $P \backslash X$ is increasing, there would exist $z_{1} \in P \backslash X$ such that $\ell\left(x, z_{1}\right)=n+1$ and so $\ell(x,(P \backslash X)) \leq n+1$. Thus $x, z \in \operatorname{Max}(P)$ and $x>y$, for some $y$ such that $\ell(y,(P \backslash X))=n+1$. So $x \in\left[X^{(n+1)(* *) \prime}\right)$, which is a contradiction. Thus $X^{(n+2)(\prime *)} \subseteq\{x: \ell(x,(P \backslash X))>n+2\}$ and we conclude that $X^{(n+2)(*)}=\{x: \ell(x,(P \backslash X))>n+2\}$. 
Let $P$ be a $p m$-space and $x, y \in P$. The $\zeta$-distance from $x$ to $y$, denoted $\ell_{\zeta}(x, y)$, is the least element of the set $\{\ell(x, y), \ell(x, \zeta(y))\}$, that is, $\ell_{\zeta}(x, y)=\min \{\ell(x, y), \ell(x, \zeta(y))\}$. Since $\ell(x, \zeta(y))=\ell(\zeta(x), y)$, we have that $\ell_{\zeta}(x, y)=\ell_{\zeta}(y, x)$ and we just call $\ell_{\zeta}(x, y)$ the $\zeta$-distance between $x$ and $y$. If $\ell_{\zeta}(x, y)=\infty$, we say that the $\zeta$-distance between $x$ and $y$ is infinite, otherwise it is said to be finite.

From the definition of $\zeta$-distance and from the fact that $\zeta$ is an involutive dual order-isomorphism, it is clear that $\ell_{\zeta}(x, y)=\ell_{\zeta}(\zeta(x), \zeta(y))=\ell_{\zeta}(x, \zeta(y))=\ell_{\zeta}(\zeta(x), y)$.

Let $n<\omega$. We say that $P$ is of $\zeta$-width $n$ if, for all $x, y \in P$, whenever it is the case that $\ell_{\zeta}(x, y)$ is finite, then $\ell_{\zeta}(x, y) \leq n$. It is clear that $P$ is of $\zeta$-width $n$ if and only if, for any order component $Q \subseteq P$ and any $x, y \in Q, \ell_{\zeta}(x, y) \leq n$.

The immediate objective is to establish Theorem 3.4 the next lemma is in preparation for this.

LEMMA 3.3. Let $P$ be a pm-space of height at most $1, X \subseteq P$ a clopen decreasing set and let $Q$ be an order component of $P$. We have

(1) If $Q \cup \zeta(Q) \subseteq X$, then $Q \cup \zeta(Q) \subseteq\left(X \cap X^{\prime *}\right)^{m(*)}$, for every $m<\omega$.

(2) If $(Q \cup \zeta(Q)) \cap X=\emptyset$, then $(Q \cup \zeta(Q)) \cap\left(X \cap X^{\prime *}\right)^{m(\prime *)}=\emptyset$, for every $m<\omega$.

(3) If $(Q \cup \zeta(Q)) \cap X \neq \emptyset,(Q \cup \zeta(Q)) \cap(P \backslash X) \neq \emptyset$ and there exists $n<\omega$, such that, for any $x, y \in Q, \ell_{\zeta}(x, y) \leq n$, then $(Q \cup \zeta(Q)) \cap\left(X \cap X^{\prime *}\right)^{m(*)}=\emptyset$, for $n \leq m<\omega$.

Proof. Recall that $\zeta$ is an involutive dual order-isomorpism. Let $Q \subseteq P$ be an order component. Then $\zeta(Q)$ is also an order component.

(1) It is sufficient to show that for any clopen decreasing set $Y \subseteq P$ such that $Q \cup \zeta(Q) \subseteq Y$, we have $Q \cup \zeta(Q) \subseteq Y^{*}$. Let $x \in Q \cup \zeta(Q)$. We have that $Q_{x}=Q$ or $Q_{x}=\zeta(Q)$, so $Q_{x} \subseteq Q \cup \zeta(Q) \subseteq Y$ and, consequently, $\zeta\left(Q_{x}\right) \subseteq Q \cup \zeta(Q) \subseteq Y$. Applying Lemma 3.2, we conclude first that $\ell(x, \zeta(P \backslash Y))$ is infinite and then that $x \in Y^{\prime *}$. Thus $Q \cup \zeta(Q) \subseteq Y^{\prime *}$.

(2) Suppose that $(Q \cup \zeta(Q)) \cap X=\emptyset$. Then $(Q \cup \zeta(Q)) \cap\left(X \cap X^{\prime *}\right)=\emptyset$. As, for any $m<\omega$, $\left(X \cap X^{\prime *}\right)^{m(*)} \subseteq X \cap X^{\prime *}$, we conclude that $(Q \cup \zeta(Q)) \cap\left(X \cap X^{\prime *}\right)^{m(*)}=\emptyset$, for every $m<\omega$.

(3) Suppose that $(Q \cup \zeta(Q)) \cap X \neq \emptyset,(Q \cup \zeta(Q)) \cap(P \backslash X) \neq \emptyset$ and that there exists $n<\omega$, such that, for any $x, y \in Q, \ell_{\zeta}(x, y) \leq n$. As $\ell_{\zeta}(x, y)=\ell_{\zeta}(\zeta(x), \zeta(y))=\ell_{\zeta}(x, \zeta(y))=\ell_{\zeta}(\zeta(x), y)$, we have that $\ell_{\zeta}(x, y) \leq n$, for any $x, y \in Q \cup \zeta(Q)$. Take $y \in(Q \cup \zeta(Q)) \cap(P \backslash X)$. As $y \in P \backslash X$, we have $\zeta(y) \in \zeta(P \backslash X)$. Applying Lemma 3.2, we know that $\zeta(y) \notin X^{\prime *}$, since $\ell(\zeta(y), \zeta(P \backslash X))=0$. In particular $y, \zeta(y) \notin X \cap X^{\prime *}$. That is $y, \zeta(y) \in P \backslash\left(X \cap X^{\prime *}\right)$ and, likewise, $y, \zeta(y) \in \zeta\left(P \backslash\left(X \cap X^{\prime *}\right)\right)$. Let $x \in Q \cup \zeta(Q)$. As $y \in Q \cup \zeta(Q)$, we have $\ell_{\zeta}(x, y) \leq n$, that is, $\ell(x, y) \leq n$ or $\ell(x, \zeta(y)) \leq n$. As $y, \zeta(y) \in P \backslash\left(X \cap X^{\prime *}\right)$, we conclude that $\ell\left(x,\left(P \backslash\left(X \cap X^{\prime *}\right)\right)\right) \leq n$ and, as $y, \zeta(y) \in \zeta\left(P \backslash\left(X \cap X^{\prime *}\right)\right)$, we have that $\ell\left(x, \zeta\left(P \backslash\left(X \cap X^{\prime *}\right)\right)\right) \leq n$. Applying Lemma 3.2, $x \notin\left(X \cap X^{\prime *}\right)^{n(1 *)}$. That is, $(Q \cup \zeta(Q)) \cap\left(X \cap X^{\prime *}\right)^{n(*)}=\emptyset$ and the result follows from the fact that, for any $m \geq n$, $\left(X \cap X^{\prime *}\right)^{m(/ *)} \subseteq\left(X \cap X^{\prime *}\right)^{n(/ *)}$.

THEOREM 3.4. Let $L$ be a regular pm-algebra and $P$ be its dual pm-space. If $P$ has $\zeta$-width $n<\omega$, then $L$ is of range $n$.

Proof. As $L$ is a regular $p m$-algebra, $P$ has height at most 1, by Theorem 3.1 Suppose the $\zeta$ width of $P$ is $n<\omega$. If $P=\emptyset$, then $L$ is trivial and is, obviously, of range $n$. Let $P \neq \emptyset$. We must show that, for any $x \in L,\left(x \wedge x^{\prime *}\right)^{n(\prime *)}=\left(x \wedge x^{\prime *}\right)^{(n+1)(/ *)}$, or, equivalently, for any clopen decreasing set $X \subseteq P,\left(X \cap X^{\prime *}\right)^{n(/ *)}=\left(X \cap X^{\prime *}\right)^{(n+1)(/ *)}$. As the $\zeta$-width of $P$ is $n$, 
then, for any order component $Q \subseteq P$ and any $x, y \in Q, \ell_{\zeta}(x, y) \leq n$. Let $X \subseteq P$ be a clopen decreasing set. For any order component $Q$ we have that $Q \cup \zeta(Q) \subseteq X$, or $(Q \cup \zeta(Q)) \cap X=\emptyset$, or $(Q \cup \zeta(Q)) \cap X \neq \emptyset$ and $(Q \cup \zeta(Q)) \cap(P \backslash X) \neq \emptyset$, so, applying, respectively, (1), (2) and (3) of the previous lemma 3.3, we conclude that $(Q \cup \zeta(Q)) \cap\left(X \cap X^{\prime *}\right)^{n(*)}=(Q \cup \zeta(Q)) \cap\left(X \cap X^{\prime *}\right)^{(n+1)()^{(*)} \text {. }}$. Thus $\left(X \cap X^{\prime *}\right)^{n(*)}=\left(X \cap X^{\prime *}\right)^{(n+1)(/ *)}$, since the order components of $P$ form a partition of $P$.

The next objective is the converse of Theorem 3.4, for which (3) of the next lemma isolates the technical step needed.

Let $P$ be $p m$-space. For each $n<\omega$, and each $x \in P$, let $D_{n}(x):=\{z \in P: \ell(z, x) \leq n\}$.

LEMMA 3.5. Let $P$ be a pm-space of height at most $1, x, y \in P$ and $n<\omega$. We have

(1) If $x \in \operatorname{Min}(P)$, then $D_{n}(x)$ is closed and decreasing whenever $n$ is even, and $D_{n}(x)$ is closed and increasing whenever $n$ is odd.

(2) If $x \in \operatorname{Max}(P)$, then $D_{n}(x)$ is closed and increasing whenever $n$ is even, and $D_{n}(x)$ is closed and decreasing whenever $n$ is odd.

(3) If $n \geq 1, \ell(x, y)=n, \ell(x, \zeta(y)) \not \leq n$ and $D_{n-1}(x) \cup D_{n}(\zeta(x))$ is decreasing, then there exists a clopen decreasing set $X$ such that $\ell\left(x,\left(P \backslash\left(X \cap X^{\prime *}\right)\right)\right)=n$.

Proof. (1) Suppose $x \in \operatorname{Min}(P)$. We have that $D_{0}(x)=\{z: \ell(z, x)=0\}=\{x\}$, which is closed and, since $x \in \operatorname{Min}(P)$, it is decreasing. We proceed inductively. Suppose that $n$ is even and that $D_{n}(x)$ is closed and decreasing. We have $D_{n+1}(x)=\{z: \ell(z, x) \leq n+1\}$. Obviously, $\left[D_{n}(x)\right) \subseteq D_{n+1}(x)$. Let $z \in D_{n+1}(x)$. Then, either $z \in D_{n}(x)$ or $\ell(z, x)=n+1$. In the latter case, as $n+1$ is odd and $x \in \operatorname{Min}(P)$, we have that $z \in \operatorname{Max}(P)$ and so $z>z_{1}$, for some $z_{1} \in D_{n}(x)$. Thus, $z \in\left[D_{n}(x)\right)$. Consequently, $D_{n+1}(x)=\left[D_{n}(x)\right)$, which is increasing and closed, since $D_{n}(x)$ is closed. Now, it is obvious that $\left(D_{n+1}(x)\right] \subseteq D_{n+2}(x)$. Let $z \in D_{n+2}(x)$. Then, either $z \in D_{n+1}(x)$ or $\ell(z, x)=n+2$. In the latter case, as $n+2$ is even and $x \in \operatorname{Min}(P)$, we have that $z \in \operatorname{Min}(P)$ and so $z<z_{1}$, for some $z_{1} \in D_{n+1}(x)$. Thus, $z \in\left(D_{n+1}(x)\right]$. Consequently, $D_{n+2}(x)=\left(D_{n+1}(x)\right]$, which is decreasing and closed, since $D_{n+1}(x)$ is closed.

(2) Suppose $x \in \operatorname{Max}(P)$. Then $\zeta(x) \in \operatorname{Min}(P)$. As $\zeta$ is an involutive continuous dual orderisomorphism $\zeta\left(D_{n}(\zeta(x))\right)=D_{n}(x)$, for any $n<\omega$, and $\zeta\left(D_{n}(\zeta(x))\right)$ is closed and decreasing (respectively, increasing) if and only if $D_{n}(\zeta(x))$ is closed and increasing (respectively, decreasing). Now we just apply (1).

(3) Suppose $n \geq 1, \ell(x, y)=n, \ell(x, \zeta(y)) \not \leq n$ and $D_{n-1}(x) \cup D_{n}(\zeta(x))$ is decreasing. By (1) and (2), we know that $D_{n-1}(x) \cup D_{n}(\zeta(x))$ is closed. As $\ell(x, y)=n$ and $\ell(y, \zeta(x))=\ell(x, \zeta(y)) \not \leq n$, we have that $y \notin D_{n-1}(x) \cup D_{n}(\zeta(x))$. So, the set $D_{n-1}(x) \cup D_{n}(\zeta(x))$ is closed and decreasing and $y \notin D_{n-1}(x) \cup D_{n}(\zeta(x))$. It follows that there exists a clopen increasing set $Y$ such that $y \in Y$ and $\left(D_{n-1}(x) \cup D_{n}(\zeta(x))\right) \cap Y=\emptyset$. Let $X=P \backslash Y$, which is a clopen decreasing set such that $D_{n-1}(x) \cup D_{n}(\zeta(x)) \subseteq X$ and $y \notin X$. From $D_{n}(\zeta(x)) \cap Y=\emptyset$, we get $\zeta\left(D_{n}(\zeta(x))\right) \cap \zeta(Y)=\emptyset$. Therefore, as $\zeta\left(D_{n}(\zeta(x))\right)=D_{n}(x)$, it follows that $D_{n}(x) \cap \zeta(Y)=\emptyset$. Suppose $D_{n-1}(x) \nsubseteq X^{\prime *}$, that is, there exists $z \in D_{n-1}(x)$ such that $z \notin X^{\prime *}$. For such a $z$ we have $\ell(z, x) \leq n-1$ since $z \in D_{n-1}(x)$ and, applying Lemma 3.2. $\ell(z, \zeta(Y)) \leq 1$ since $z \notin X^{\prime *}$. Consequently, $\ell(x, \zeta(Y)) \leq n$ and $D_{n}(x) \cap \zeta(Y) \neq \emptyset$, a contradiction. Thus $D_{n-1}(x) \subseteq X^{\prime *}$ and so $D_{n-1}(x) \subseteq X \cap X^{\prime *}$. As $y \notin X \cap X^{\prime *}$ and $\ell(x, y)=n$, we have $\ell\left(x,\left(P \backslash\left(X \cap X^{\prime *}\right)\right)\right) \leq n$. But $D_{n-1}(x) \subseteq X \cap X^{\prime *}$ and, consequently, $\ell\left(x,\left(P \backslash\left(X \cap X^{\prime *}\right)\right)\right)>n-1$. So $\ell\left(x,\left(P \backslash\left(X \cap X^{\prime *}\right)\right)\right)=n$, as required.

THEOREM 3.6. Let $L$ be a regular pm-algebra and $P$ be its dual pm-space. If there exist $x, y \in P$ such that $\ell_{\zeta}(x, y)=n$ for some $1 \leq n<\omega$, then $L$ does not satisfy the identity $\left(x \wedge x^{\prime *}\right)^{(n-1)(* *)} \approx$ $\left(x \wedge x^{\prime *}\right)^{n(\prime)}$. 
Proof. As $L$ is a regular $p m$-algebra, by Theorem 3.1, $P$ has height at most 1 . Suppose $\ell_{\zeta}(x, y)=n$ for some $x, y \in P$ and $n \geq 1$. We begin with two small observations that will simplify the subsequent argument. First, we recall that $\ell_{\zeta}(x, y)=\ell_{\zeta}(\zeta(x), \zeta(y))$. Second, suppose that $\ell_{\zeta}(x, y)=\ell(x, \zeta(y))$. Since $\ell_{\zeta}(\zeta(x), y)=\ell_{\zeta}(x, y)$, we have $\ell_{\zeta}(\zeta(x), y)=\ell(x, \zeta(y))=\ell(\zeta(x), y)$. Thus, with no loss of generality, we will assume, by the first observation, that $y \in \operatorname{Max}(P)$ and, by the second, that $\ell_{\zeta}(x, y)=\ell(x, y)$.

Suppose $n$ is odd. Since $y \in \operatorname{Max}(P)$ and $n=\ell_{\zeta}(x, y)=\ell(x, y)$, we have that $x \in \operatorname{Min}(P)$ and so $\zeta(x) \in \operatorname{Max}(P)$. We know, by (1) and (2) of Lemma 3.5 that both $D_{n-1}(x)$ and $D_{n}(\zeta(x))$ are closed and decreasing and so $D_{n-1}(x) \cup D_{n}(\zeta(x))$ is closed and decreasing. Since $\ell_{\zeta}(x, y)=n$, we have that $\ell(x, \zeta(y)) \not \leq n-1$. As $x, \zeta(y) \in \operatorname{Min}(P)$ and $n$ is odd we cannot have $\ell(x, \zeta(y))=n$. So $\ell(x, \zeta(y)) \not \leq n$. Applying (3) of Lemma 3.5, there exists a clopen decreasing set $X$ such that $\ell\left(x,\left(P \backslash\left(X \cap X^{\prime *}\right)\right)\right)=n$. For such an $X$, applying Lemma 3.2 we have that $x \in\left(X \cap X^{\prime *}\right)^{(n-1)(/ *)}$, whilst $x \notin\left(X \cap X^{\prime *}\right)^{(n+1)(/ *)}$. In particular, $\left(X \cap X^{\prime *}\right)^{(n-1)(1 *)} \neq\left(X \cap X^{\prime *}\right)^{(n+1)(/ *)}$. It follows that $\left(X \cap X^{\prime *}\right)^{(n-1)(/ *)} \neq\left(X \cap X^{\prime *}\right)^{n(/ *)}$, that is $L$ does not satisfy the identity $\left(x \wedge x^{\prime *}\right)^{(n-1)(/ *)} \approx$ $\left(x \wedge x^{\prime *}\right)^{n(\prime *)}$.

Now suppose $n$ is even. Since $n=\ell_{\zeta}(x, y)=\ell(x, y)$ and $y \in \operatorname{Max}(P)$, we have that $x \in \operatorname{Max}(P)$ and so $\zeta(x) \in \operatorname{Min}(P)$. We know, by (1) and (2) of Lemma 3.5, that both $D_{n-1}(x)$ and $D_{n}(\zeta(x))$ are closed and decreasing and so $D_{n-1}(x) \cup D_{n}(\zeta(x))$ is closed and decreasing. Since $\ell_{\zeta}(x, y)=n$, we have that $\ell(x, \zeta(y)) \not \leq n-1$. As $x \in \operatorname{Max}(P)$ and $\zeta(y) \in \operatorname{Min}(P)$ and $n$ is even we cannot have $\ell(x, \zeta(y))=n$. So $\ell(x, \zeta(y)) \not \leq n$. Applying (3) of Lemma 3.5. there exists a clopen decreasing set $X$ such that $\ell\left(x,\left(P \backslash\left(X \cap X^{\prime *}\right)\right)\right)=n$. For such an $X$, we have $\ell\left(\zeta(x), \zeta\left(P \backslash\left(X \cap X^{\prime *}\right)\right)\right)=n$. Therefore, by Lemma 3.2. $\zeta(x) \in\left(X \cap X^{\prime *}\right)^{(n-1)(* *)}$, whilst $\zeta(x) \notin\left(X \cap X^{\prime *}\right)^{(n+1)(/ *)}$. In particular, $\left(X \cap X^{\prime *}\right)^{(n-1)(/ *)} \neq\left(X \cap X^{\prime *}\right)^{(n+1)(/ *)}$. It follows that $\left(X \cap X^{\prime *}\right)^{(n-1)(/ *)} \neq\left(X \cap X^{\prime *}\right)^{n(\prime *)}$, that is $L$ does not satisfy the identity $\left(x \wedge x^{\prime *}\right)^{(n-1)(/ *)} \approx\left(x \wedge x^{\prime *}\right)^{n(/ *)}$.

COROLLARY 3.7. Let $L$ be a regular pm-algebra and $P$ be its dual pm-space. Then $L$ is of range $n<\omega$ if and only if $P$ is of $\zeta$-width $n$.

Proof. Suppose $L$ is of range $n$. Suppose $x, y \in P$ such that $\ell_{\zeta}(x, y)$ is finite. If $\ell_{\zeta}(x, y)=k>n$, then, by Theorem [3.6. $L$ does not satisfy the identity $\left(x \wedge x^{\prime *}\right)^{\left.(k-1)()^{\prime *}\right)} \approx\left(x \wedge x^{\prime *}\right)^{k(*)}$ and, as $k-1 \geq n, L$ is not of range $n$, a contradiction. So, $\ell_{\zeta}(x, y) \leq n$. Thus, $P$ is of $\zeta$-width $n$. For the converse just apply Theorem 3.4 .

\section{Subdirectly irreducible algebras and local finiteness}

As we have already mentioned, for each $n<\omega$, the variety $\mathbf{M}_{n}$ of regular $p m$-algebras of range $n$ is a discriminator variety and, consequently, its sudirectly irreducible algebras are the simple ones. We begin by characterising the simple algebras of $\mathbf{M}_{n}, n<\omega$, via their dual spaces. For this purpose, the following theorem will be useful.

THEOREM 4.1. Let $L \in \mathbf{M}_{n}$ and $P$ be its dual pm-space. Then all order components of $P$ are closed.

Proof. Suppose that $L \in \mathbf{M}_{n}$. Then, by Corollary 3.7, $P$ is of $\zeta$-width $n$. So, for all $x, y \in P$ such that $\ell_{\zeta}(x, y)$ is finite, we have $\ell_{\zeta}(x, y) \leq n$.

Let $Q$ be an order component of $P$ and $x \in Q$. Obviously, $D_{n}(x) \subseteq Q$. We know that $Q \cap \zeta(Q)=\emptyset$ or $Q=\zeta(Q)$. So, we have two cases to consider. Firstly, suppose that $Q \cap \zeta(Q)=\emptyset$. 
Let $y \in Q$. We have that $\ell(x, y)$ is finite and $\ell(x, \zeta(y))$ is infinite, so $\ell(x, y)=\ell_{\zeta}(x, y) \leq n$ and, consequently, $y \in D_{n}(x)$. Thus, $Q=D_{n}(x)$, which is closed in $P$, by (1) and (2) of Lemma 3.5. Secondly, suppose that $Q=\zeta(Q)$. Consider $D_{n}(x)$ and $D_{n}(\zeta(x))$. Obviously, $D_{n}(x) \subseteq Q$ and, since $\zeta(x) \in Q$, we also have $D_{n}(\zeta(x)) \subseteq Q$. Thus, $D_{n}(x) \cup D_{n}(\zeta(x)) \subseteq Q$. Let $y \in Q$. As $Q=\zeta(Q)$, we have that both $\ell(y, x)$ and $\ell(y, \zeta(x))$ are finite and, since $\ell_{\zeta}(y, x) \leq n$, at least, one of them does not exceed $n$. Thus, $y \in D_{n}(x) \cup D_{n}(\zeta(x))$ and we have $Q=D_{n}(x) \cup D_{n}(\zeta(x))$. By (1) and (2) of Lemma 3.5, $Q$ is closed in $P$.

THEOREM 4.2. Let $L \in \mathbf{M}_{n}$ and $P$ be its dual pm-space. Then the following are equivalent:

(1) $L$ is simple,

(2) $P=Q \cup \zeta(Q)$ where $Q$ is an order component of $P$.

Proof. As we have already observed in Section 2 there is a lattice-isomorphism from the lattice of congruences on $L$ to the lattice of open sets $X$ of $P$ such that $\zeta(X)=X$ and $[\operatorname{Min}(P) \cap X) \subseteq X$. Moreover any such open set also satisfies $(\operatorname{Max}(P) \cap X] \subseteq X$.

Suppose that $L$ is simple. Consider $Q \cup \zeta(Q)$, where $Q$ is an order component of $P$. Suppose $P \neq Q \cup \zeta(Q)$. Let $X=P \backslash(Q \cup \zeta(Q))$. We wish to show that $X$ represents a congruence on $P$. Observe that $X$ is an open set, since both $Q$ and $\zeta(Q)$ are order components and hence closed, by the previous theorem. Obviously, $\zeta(X)=X$. Let $y \in[\operatorname{Min}(P) \cap X)$. Then $y \geq x$, for some $x \in \operatorname{Min}(P) \cap X$. If $y \in Q \cup \zeta(Q)$, so would $x$, since $Q$ and $\zeta(Q)$ are order components. So $y \in P \backslash(Q \cup \zeta(Q))=X$. Thus $[\operatorname{Min}(P) \cap X) \subseteq X$ and $X$ represents a congruence on $L$. Since $X \neq \emptyset$ and $X \neq P$, we have a contradiction to the fact that $L$ is simple.

Conversely, suppose that $P=Q \cup \zeta(Q)$ for an order component $Q$. Let $\theta$ be a congruence on $L$ different from the equality congruence and let $X$ be the open set that represents $\theta$. Then $X \neq \emptyset, \zeta(X)=X,[\operatorname{Min}(P) \cap X) \subseteq X$ and $(\operatorname{Max}(P) \cap X] \subseteq X$. Since $P=\operatorname{Min}(P) \cup \operatorname{Max}(P)$, we conclude that $X$ is increasing and decreasing. From $\zeta(X)=X \neq \emptyset$ and $P=Q \cup \zeta(Q)$, we have that $\emptyset \neq X=(Q \cap X) \cup(\zeta(Q) \cap X)=(Q \cap X) \cup(\zeta(Q) \cap \zeta(X))=(Q \cap X) \cup \zeta(Q \cap X)$ and hence $Q \cap X \neq \emptyset$. Thus $Q \subseteq X$, since $X$ is both increasing and decreasing, and we conclude that $P=Q \cup \zeta(Q) \subseteq X \cup \zeta(X)=X$. So $\theta$ is the universal congruence.

COROLLARY 4.3. Let $L$ be a non-trivial regular pm-algebra and $P$ be its dual pm-space. Then $L$ is a simple algebra of $\mathbf{M}_{n}$ if and only if $\ell(x, y) \leq n$ or $\ell(x, \zeta(y)) \leq n$, for all $x, y \in P$.

Proof. Suppose $L$ is a simple algebra of $\mathbf{M}_{n}$. Then, by Corollary 3.7 and Theorem $4.2 P$ is of $\zeta$-width $n$ and $P=Q \cup \zeta(Q)$ with $Q$ order component. The set $\zeta(Q)$ is also an order component. Let $x, y \in P$. If $x, y \in Q$ or $x, y \in \zeta(Q)$, then $\ell(x, y)$ is finite. Otherwise $\ell(x, \zeta(y))$ is finite, since both elements will belong to the same order component. Either way $\ell_{\zeta}(x, y)$ is finite and then, as $P$ is of $\zeta$-width $n, \ell_{\zeta}(x, y) \leq n$, that is $\ell(x, y) \leq n$ or $\ell(x, \zeta(y)) \leq n$.

Conversely, suppose that, for all $x, y \in P, \ell(x, y) \leq n$ or $\ell(x, \zeta(y)) \leq n$. It is obvious that $P$ is of $\zeta$-width $n$ and then $L \in \mathbf{M}_{n}$, by Corollary 3.7 Let $x \in P$ and let $Q_{x}$ be the order component such that $x \in Q_{x}$. For any $y \in P$, we have $\ell(x, y) \leq n$ or $\ell(x, \zeta(y)) \leq n$ and, consequently, $y \in Q_{x}$ or $y \in \zeta\left(Q_{x}\right)$. Thus $P=Q_{x} \cup \zeta\left(Q_{x}\right)$ and $L$ is simple, by the previous theorem.

In Figure 1, clouds denote order components.

The next theorem gives a description, via their dual spaces, of the non-trivial algebras in $\mathbf{M}_{0}$, leading to the description of its simple algebras presented in Corollary 4.5 . 


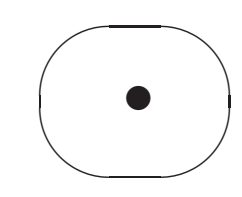

$Q_{0}$

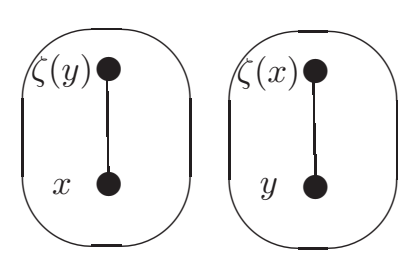

$Q_{3}$

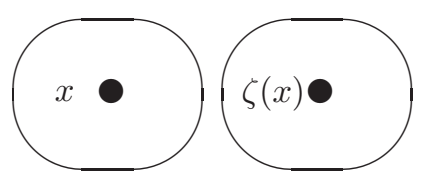

$Q_{1}$

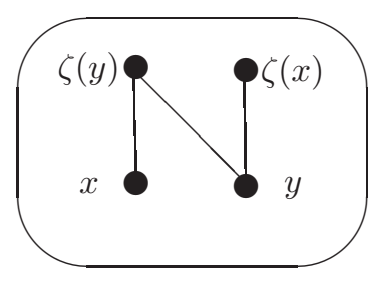

$Q_{4}$

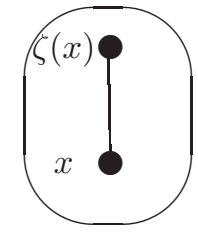

$Q_{2}$

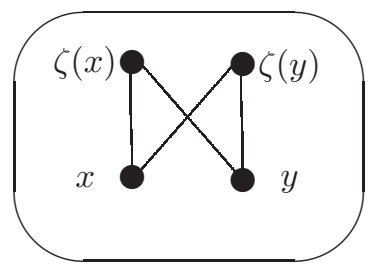

$Q_{5}$

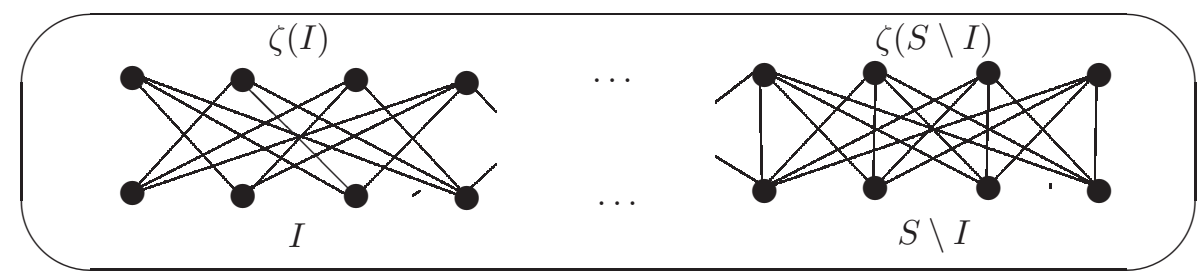

$Q_{6}(I, S)$

Figure 1

THEOREM 4.4. Let $L$ be a non-trivial pm-algebra and $P$ be its dual pm-space. Then $L \in \mathbf{M}_{0}$ if and only if, for every order component $Q \subseteq P, Q \cup \zeta(Q)$ is a copy of one of $Q_{0}, Q_{1}$, or $Q_{2}$ (as diagrammed in Figure 1).

Proof. Applying Theorem 3.1 and Corollary 3.7 we know that $L \in \mathbf{M}_{0}$ if and only $P$ has height at most 1 and, for any order component $Q$ of $P$ and any $x, y \in Q$, we have $\ell_{\zeta}(x, y)=0$. Suppose $L \in \mathbf{M}_{0}$. Let $Q$ be an order component of $P$ and $x \in Q$. If $y \in Q$ and $y \neq x$, then, as $\ell_{\zeta}(y, x)=0$, we have that $\ell(y, \zeta(x))=0$, that is $y=\zeta(x)$. So $|Q| \leq 2$, since $\zeta$ is a bijection. Now it is clear that $Q \cup \zeta(Q)$ is a copy of of $Q_{0}$ or $Q_{1}$, if $|Q|=1$, whilst $Q \cup \zeta(Q)$ is a copy of $Q_{2}$, if $|Q|=2$. The converse is straightforward.

In [22, Theorem 6.5], Sankappanavar described, up to isomorphism, the 6 subdirectly irreducible pseudocomplemented de Morgan algebras of range 0 . Exactly 3 of them are regular. So $\mathbf{M}_{0}$ has, up to isomorphism, 3 simple algebras. Their dual spaces are copies of $Q_{0}, Q_{1}$, or $Q_{2}$ as diagrammed in Figure 1. This fact is also an immediate consequence of Theorems 4.2 and 4.4

COROLLARY 4.5. The simple algebras in $\mathbf{M}_{0}$ are the ones whose dual pm-space is a copy of $Q_{0}, Q_{1}$, or $Q_{2}$ (as diagrammed in Figure 1 ).

Notice that the simple algebras in $\mathbf{M}_{0}$ are just the simple de Morgan algebras with the inherent 
pseudocomplementation.

For a Stone space $(S ; \tau)$ and a subset $I$ of the set of its isolated points, let $Q_{6}(I, S)$ be the quadruple $(S \cup \zeta(S) ; \tau, \leq, \zeta)$ where $3 \leq|S|, \zeta(S)$ denotes a homeomorphic copy of $S, \tau$ is the union topology, $\zeta(\zeta(x))=x$ for $x \in S$, and $\leq$ is the partial order on $S \cup \zeta(S)$ induced by, for $x, y \in S$,

$$
x<\zeta(y) \text { iff } x \neq y \text { or } x \notin I .
$$

THEOREM 4.6. The quadruple $Q_{6}(I, S)=(S \cup \zeta(S) ; \tau, \leq, \zeta)$ is a pm-space of height 1 .

Proof. It is immediate that $(S \cup \zeta(S) ; \leq)$ has height 1 and that $(S \cup \zeta(S) ; \tau)$ is a compact Hausdorff space. Moreover, whenever $z$ is an isolated point of the Stone space $S$ or of the Stone space $\zeta(S), z$ is also an isolated point of $Q_{6}(I, S)$ and, consequently, $\{z\}$ is clopen in $Q_{6}(I, S)$ and so are $S \backslash\{z\}$ and $\zeta(S) \backslash\{z\}$.

Let $x, y \in S \cup \zeta(S)$ be such that $x \not \leq y$. First, suppose $x \in S$. Then either $y \in S$ and there is a set $X \subseteq S$ clopen in $S$, and so clopen in $Q_{6}(I, S)$ and necessarily decreasing, such that $y \in X$ and $x \notin X$, or else $y=\zeta(x)$ with $x \in I$. In this latter case, namely $y=\zeta(x), S \backslash\{x\}$ and $\{\zeta(x)\}$ are clopen in $Q_{6}(I, S)$, since $x$ and $\zeta(x)$ are isolated points. Thus $\{\zeta(x)\} \cup(S \backslash\{x\})$ is clopen decreasing in $Q_{6}(I, S)$ and $x \notin\{\zeta(x)\} \cup(S \backslash\{x\})$. Next, suppose $x \in \zeta(S)$. Then, since $S$ is clopen decreasing in $Q_{6}(I, S)$ and $x \notin S$, the case $y \in S$ is obvious, and if $y \in \zeta(S)$, there is a set $X \subseteq \zeta(S)$ clopen in $\zeta(S)$, and so in $Q_{6}(I, S)$, such that $y \in X$ and $x \notin X$ from which $y \in X \cup S$, $x \notin X \cup S$ and $X \cup S$ is a clopen decreasing set of $Q_{6}(I, S)$.

Thus, $(S \cup \zeta(S) ; \tau, \leq)$ is a Priestley space and it is clear that $\zeta$ is a continuous order-reversing involution.

Suppose $X \subseteq S \cup \zeta(S)$ is a clopen decreasing set. Obviously, if $X=\emptyset$, then $[X)=\emptyset$. If $X=\{x\}$ for some $x \in I$, then $[X)=\{x\} \cup(\zeta(S) \backslash\{\zeta(x)\})$ which is clopen in $Q_{6}(I, S)$, since $x$ and $\zeta(x)$ are isolated points. In the remaining cases for $X$, if $|X \cap S| \geq 2$, or $X=\{x\}$ for some $x \in S \backslash I$, it is obvious that $[X)=X \cup \zeta(S)$. Observe that whenever $X \cap \zeta(S) \neq \emptyset$, then, as $X$ is decreasing, $|X \cap S| \geq 2$, and, as already argued, $[X)=X \cup \zeta(S)$. So whatever the case, we always have $[X)=X \cup \zeta(S)$, which is clearly clopen in $Q_{6}(I, S)$.

Therefore $(S \cup \zeta(S) ; \tau, \leq, \zeta)$ is a $p m$-space.

Our next goal is to present a description, via their dual spaces, of the non-trivial $p m$-algebras in $\mathbf{M}_{1}$, which will lead to the description of its simple algebras in Corollary 4.9.

LEMMA 4.7. Let $P$ be a pm-space of height at most 1 and $Q \subseteq P$ be an order component. Then

(1) $\operatorname{Min}(Q)=\operatorname{Min}(P) \cap Q$ and it is the set of the minimal elements of $Q$.

(2) $\operatorname{Max}(Q)=\operatorname{Max}(P) \cap Q$ and it is the set of the maximal elements of $Q$.

(3) $Q=\operatorname{Min}(Q) \cup \operatorname{Max}(Q)$, and $\operatorname{Min}(Q) \cap \operatorname{Max}(Q) \neq \emptyset$ if and only if $|Q|=1$.

(4) If $|Q|>1$, then $x \neq \zeta(x)$, for any $x \in Q$.

(5) If $P$ is of $\zeta$-width 1, then $|\operatorname{Min}(Q)|=|\operatorname{Max}(Q)|$ and, if $|\operatorname{Min}(Q)|=|\operatorname{Max}(Q)|>1$, then $Q=\zeta(Q)$.

(6) If $P$ is of $\zeta$-width 1 and $|Q|>2$, then $Q=\zeta(Q)$, $|\operatorname{Min}(Q)| \geq 2$ and, for distinct $x, y$ in $\operatorname{Min}(Q)$, $x<\zeta(y)$.

Proof. (1)-(2) By definition $\operatorname{Min}(Q)=\operatorname{Min}(P) \cap(Q]$ and $\operatorname{Max}(Q)=\operatorname{Max}(P) \cap[Q)$ and the result follows immediately from the fact that $Q$ is an order component. 
(3) Immediate from (1) and (2) and the facts that $P$ is of height at most 1 and $Q$ is an order component.

(4) If $x \in Q$ is such that $x=\zeta(x)$, then $x \in \operatorname{Min}(Q) \cap \operatorname{Max}(Q)$ and, by (3), $|Q|=1$.

(5) Suppose $P$ is of $\zeta$-width 1 . We know that $|\operatorname{Min}(Q)| \geq 1$ and $|\operatorname{Max}(Q)| \geq 1$. Suppose $|\operatorname{Min}(Q)|>1$ or $|\operatorname{Max}(Q)|>1$. Then there exist $x, y \in Q$ such that $\ell(x, y) \geq 2$. Since $\ell_{\zeta}(x, y) \leq 1$, it follows that $\ell(x, \zeta(y)) \leq 1$ and then $\zeta(y) \in Q \cap \zeta(Q)$. So $Q=\zeta(Q)$ and, consequently, for every $z \in P, z \in \operatorname{Min}(Q)$ if and only if $\zeta(z) \in \operatorname{Max}(Q)$. Thus $|\operatorname{Min}(Q)|=|\operatorname{Max}(Q)|$.

(6) Suppose $P$ is of $\zeta$-width 1 and $|Q|>2$. As, by (3), $Q=\operatorname{Min}(Q) \cup \operatorname{Max}(Q)$ and by (5), $|\operatorname{Min}(Q)|=|\operatorname{Max}(Q)|$, we have that $|\operatorname{Min}(Q)| \geq 2$. That $Q=\zeta(Q)$ follows from (5). Let $x, y$ be distinct elements in $\operatorname{Min}(Q)$. Then $\ell(x, y) \geq 2$ and, consequently $\ell(x, \zeta(y)) \leq 1$. Since $|Q|>2$ and $x \in \operatorname{Min}(Q)$ and $\zeta(y) \in \operatorname{Max}(Q)$, we have that $x \neq \zeta(y)$ and, consequently, $\ell(x, \zeta(y))=1$ and hence $x<\zeta(y)$.

THEOREM 4.8. Let $L$ be a non-trivial pm-algebra and $P$ be its dual pm-space. Then $L \in \mathbf{M}_{1}$ if and only if, for every order component $Q \subseteq P, Q \cup \zeta(Q)$ is a copy of one of $Q_{0}, Q_{1}, Q_{2}, Q_{3}, Q_{4}$, $Q_{5}$, or is of type $Q_{6}(I, S)$ for some Stone space $(S ; \tau)$ and $I \subseteq S$ a subset of the isolated points of $S$ with $3 \leq|S|$ (as diagrammed in Figure 1).

Proof. Applying Theorem 3.1 and Corollary 3.7, we know that $L \in \mathbf{M}_{1}$ if and only if $P$ has height at most 1 and, for any order component $Q$ of $P$ and any $x, y \in Q$, we have $\ell_{\zeta}(x, y) \leq 1$.

Suppose that for every order component $Q \subseteq P, Q \cup \zeta(Q)$ is a copy of one of $Q_{0}, Q_{1}, Q_{2}$, $Q_{3}, Q_{4}, Q_{5}$, or is of type $Q_{6}(I, S)$ with $3 \leq|S|$. It is obvious that $P$ has height at most 1. It is also clear that if $x, y \in Q_{i}, 0 \leq i \leq 5$, then $\ell_{\zeta}(x, y) \leq 1$. Consider $Q_{6}(I, S)$ with $3 \leq|S|$. Suppose $x, y \in S$ such that $x \neq y$. Then $x<\zeta(y)$ and so $\ell_{\zeta}(x, y)=1$. As $\ell_{\zeta}(x, y)=\ell_{\zeta}(\zeta(x), \zeta(y))=\ell_{\zeta}(x, \zeta(y))=\ell_{\zeta}(\zeta(x), y)$, we have that, for any $x, y \in Q_{6}(I, S)$, $\ell_{\zeta}(x, y) \leq 1$. So $L \in \mathbf{M}_{1}$.

Conversely, suppose $L \in \mathbf{M}_{1}$ and let $Q$ be an order component of $P$. If $|Q|=1$, then $Q \cup \zeta(Q)$ is a copy of $Q_{0}$ or $Q_{1}$.

Suppose $|Q|=2$. By (4) of the previous lemma, we know that $x \neq \zeta(x)$, for any $x \in Q$. So $Q \cup \zeta(Q)$ is a copy of $Q_{2}$ if $Q=\zeta(Q)$, whilst $Q \cup \zeta(Q)$ is a copy of $Q_{3}$ if $Q \cap \zeta(Q)=\emptyset$.

Let $|Q|>2$. Observe that, by the previous lemma, $Q$ is the disjoint union of $\operatorname{Min}(Q)$ and $\operatorname{Max}(Q), Q=\zeta(Q),|\operatorname{Max}(Q)|=|\operatorname{Min}(Q)| \geq 2$ and, for distinct $x, y$ in $\operatorname{Min}(Q), x<\zeta(y)$, each of which will hold for the remainder of the proof.

Suppose initially that $|\operatorname{Min}(Q)|=2$ and, say, $\operatorname{Min}(Q)=\{x, y\}$. Then $\operatorname{Max}(Q)=\{\zeta(x), \zeta(y)\}$. Were it the case that both $x \not \leq \zeta(x)$ and $y \not \leq \zeta(y)$, then $Q$ would fail to be an order component. Thus, either $x \leq \zeta(x)$ and $y \leq \zeta(y)$, and $Q \cup \zeta(Q)=Q$ is a copy of $Q_{5}$, or else, say, $x \not \leq \zeta(x)$ and $y \leq \zeta(y)$ and $Q \cup \zeta(Q)=Q$ is a copy of $Q_{4}$.

Suppose next that $|\operatorname{Min}(Q)| \geq 3$. We have that $\operatorname{Max}(Q)=\zeta(\operatorname{Min}(Q))$. As $L \in \mathbf{M}_{1}$, applying Theorem 4.1. we know that $Q$ is a closed set. Since $\zeta(Q)=Q$ and it is obvious that $\operatorname{Min}(x) \subseteq Q$, whenever $x \in Q$, we have that $\left(Q ; \tau_{\uparrow Q}, \leq_{\left.\right|_{Q}}, \zeta_{\uparrow Q}\right)$ is a $p m$-subspace of $P$ for which $\operatorname{Min}(Q)$ and $\operatorname{Max}(Q)$ are both closed and then clopen in $Q$, as $Q$ is the disjoint union of these sets. We have that 
$\operatorname{Min}(Q)$ endowed with the induced topology is a Stone space. As $\zeta$ is a homeomorphism, $\operatorname{Max}(Q)=$ $\zeta(\operatorname{Min}(Q))$ is a homeomorphic copy of $\operatorname{Min}(Q)$. Consider the $p m$-subspace $\left(Q ; \tau_{\uparrow_{Q}}, \leq_{\Gamma_{Q}}, \zeta_{\uparrow_{Q}}\right)$. Let $x \in \operatorname{Min}(Q)$. As observed above $x<\zeta(z)$ for any $z \in \operatorname{Min}(Q) \backslash\{x\}$. Suppose $x$ is an accumulation point of $\operatorname{Min}(Q)$. If $x \not \leq \zeta(x)$, then there exists a clopen decreasing set $X$ containing $\zeta(x)$ but not $x$. Since $x$ is an accumulation point of $\operatorname{Min}(Q), \zeta(x)$ is an accumulation point of $\operatorname{Max}(Q)$ and there exists $y \in \operatorname{Max}(Q) \cap(X \backslash\{\zeta(x)\})$. As observed above $x<\zeta(\zeta(y))=y$ and so $x \in X$, since $y \in X$ and $X$ is decreasing, a contradiction. Now consider the set $I=\{x \in \operatorname{Min}(Q): x \nless \zeta(x)\}$. The elements of this set are isolated points of $\operatorname{Min}(Q)$. Thus $Q$ is a $p m$-subspace of type $Q_{6}(I, S)$ where $S$ denotes the Stone space $\operatorname{Min}(Q)$ and $I=\{x \in \operatorname{Min}(Q): x \nless \zeta(x)\} \subseteq \operatorname{Min}(Q)$ is a set of isolated points of $\operatorname{Min}(Q)$.

The next corrollary is an immediate consequence of Theorems 4.2 and 4.8

COROLLARY 4.9. The simple algebras in $\mathbf{M}_{1}$ are the ones whose dual space is a copy of $Q_{0}$, $Q_{1}, Q_{2}, Q_{3}, Q_{4}, Q_{5}$, or is of type $Q_{6}(I, S)$ for some Stone space $(S ; \tau)$ and $I \subseteq S$ a subset of the isolated points of $S$ with $3 \leq|S|$ (as diagrammed in Figure 1).

Note that $Q_{0}, Q_{2}, Q_{5}$, and $Q_{6}(\emptyset, S)$ represent Kleene algebras, but no others do.

As every subdirectly irreducible algebra in $\mathbf{M}_{n}, n<\omega$, is simple, applying Corollaries 4.5 and 4.9. we have that $\mathbf{M}_{0}$ is generated by the pseudocomplemented de Morgan algebras $E\left(Q_{0}\right), E\left(Q_{1}\right)$, and $E\left(Q_{2}\right)$, whilst $\mathbf{M}_{1}$ is generated by the pseudocomplemented de Morgan algebras $E\left(Q_{0}\right), E\left(Q_{1}\right)$, $E\left(Q_{2}\right), E\left(Q_{3}\right), E\left(Q_{4}\right), E\left(Q_{5}\right)$, and $E\left(Q_{6}(I, S)\right)$ where $(S ; \tau)$ is a Stone space, $3 \leq|S|$, and $I \subseteq S$ is a subset of the set of isolated points of $S$.

Recall that a Stone space is finite if and only if it is discrete. Consequently, all the elements of a finite Stone space are isolated points. It is clear that, up to $p m$-isomorphism, for any $n, 3 \leq n<\omega$, and any $m, 0 \leq m \leq n$, there is exactly one $Q_{6}(I, S)$ such that $|S|=n$ and $|I|=m$. Consider such an $S$ and such an $I$, we denote by $Q_{6}(m, n)$ the $p m$-space $Q_{6}(I, S)$.

In fact, as the following theorem shows, $\mathbf{M}_{1}$ is generated by the pseudocomplemented de Morgan algebras represented by $Q_{0}, Q_{1}, Q_{2}, Q_{3}, Q_{4}, Q_{5}$, and $Q_{6}(m, n)$ where $3 \leq n<\omega$ and $0 \leq m \leq n$.

THEOREM 4.10. The variety $\mathbf{M}_{1}$ is locally finite.

Proof. Since the pseudocomplemented de Morgan algebras $E\left(Q_{0}\right), E\left(Q_{1}\right), E\left(Q_{2}\right), E\left(Q_{3}\right), E\left(Q_{4}\right)$, $E\left(Q_{5}\right)$, and $E\left(Q_{6}(I, S)\right)$ where $(S ; \tau)$ is a Stone space, $3 \leq|S|$, and $I \subseteq S$ is a subset of the set of its isolated points generate $\mathbf{M}_{1}$, it is sufficient to show that for each $N<\omega$, there exists $N^{\prime}<\omega$, depending on $N$, such that, for any set of $N$ elements in any one of the pseudocomplemented de Morgan algebras $E\left(Q_{0}\right), E\left(Q_{1}\right), E\left(Q_{2}\right), E\left(Q_{3}\right), E\left(Q_{4}\right), E\left(Q_{5}\right)$, and $E\left(Q_{6}(I, S)\right)$ where $(S ; \tau)$ is a Stone space, $|S| \geq 3$, and $I$ is a subset of the set of its isolated points, generates a subalgebra whose cardinality does not exceed $N^{\prime}$ (see Mal'cev [14, VI.14 Theorem 3]). Since $\left\{E\left(Q_{i}\right): 0 \leq i \leq 5\right\}$ is a finite set of finite algebras, it is only required to show that, for each $N<\omega$, there exists $N^{\prime}<\omega$ such that, for any set of $N$ elements in any one of the pseudocomplemented de Morgan algebras $E\left(Q_{6}(I, S)\right)$ generates a subalgebra with at most $N^{\prime}$ elements.

Consider $E\left(Q_{6}(I, S)\right)$. Recall that whenever $z$ is an isolated point of the Stone space $S$ or of the Stone space $\zeta(S), z$ is also an isolated point of $Q_{6}(I, S)$ and, consequently, $\{z\}$ is clopen in $Q_{6}(I, S)$. Let $x \in I$. Then $x$ is an isolated point of $S, \zeta(x)$ is an isolated point of $\zeta(S)$ and we have that

$$
(\zeta(x)]=\{\zeta(x)\} \cup(S \backslash\{x\}),
$$


is a clopen decreasing set of $Q_{6}(I, S)$.

Claim. Let $\mathcal{F}$ be a Boolean subalgebra of the Boolean algebra of clopen sets of $S$, let $\mathcal{F}_{I}=\{\{x\} \in$ $\mathcal{F}: x \in I\}$ and

$$
K(\mathcal{F}):=\mathcal{F} \cup\{\zeta(X) \cup S: X \in \mathcal{F}\} \cup\left\{(\zeta(x)]:\{x\} \in \mathcal{F}_{I}\right\} .
$$

Then $K(\mathcal{F})$ is a subalgebra of $E\left(Q_{6}(I, S)\right)$.

First notice that all the elements of $K(\mathcal{F})$ are clopen decreasing sets of $Q_{6}(I, S)$ and, since $\emptyset, S \in \mathcal{F}$, we have that $\emptyset \in K(\mathcal{F})$ and $S \cup \zeta(S) \in K(\mathcal{F})$.

We begin by showing that $K(\mathcal{F})$ is closed under unions and intersections. Consider the various cases, starting with those that involve the first two types of clopen decreasing sets for which there are six cases.

Let $X, Y \in \mathcal{F}$. Obviously $X \cup Y, X \cap Y \in \mathcal{F}$. Further, we have that $X \cup(\zeta(Y) \cup S)=$ $\zeta(Y) \cup S$ and $X \cap(\zeta(Y) \cup S)=X$, whilst $(\zeta(X) \cup S) \cup(\zeta(Y) \cup S)=\zeta(X \cup Y) \cup S$ and $(\zeta(X) \cup S) \cap(\zeta(Y) \cup S)=\zeta(X \cap Y) \cup S$. It remains to consider the cases involving the third type of clopen decreasing set for which there are another six cases. Let $\{x\} \in \mathcal{F}_{I}$. Then $\{x\} \in \mathcal{F}$, $x \in I$ and $S \backslash\{x\} \in \mathcal{F}$. We have $X \cap(\zeta(x)]=X \cap(\{\zeta(x)\} \cup(S \backslash\{x\}))=X \cap(S \backslash\{x\})$ and $(\zeta(X) \cup S) \cup(\zeta(x)]=\zeta(X \cup\{x\}) \cup S$. Four cases remain. First consider $X \cup(\zeta(x)]$ and $(\zeta(X) \cup S) \cap(\zeta(x)]$. If $x \notin X$, then $X \cup(\zeta(x)]=X \cup\{\zeta(x)\} \cup(S \backslash\{x\})=\{\zeta(x)\} \cup(S \backslash\{x\})=(\zeta(x)]$ and $(\zeta(X) \cup S) \cap(\zeta(x)]=S \backslash\{x\}$, whilst, if $x \in X, X \cup(\zeta(x)]=\{\zeta(x)\} \cup S=\zeta(\{x\}) \cup S$ and $(\zeta(X) \cup S) \cap(\zeta(x)]=(\zeta(x)]$. Finally, two cases remain, each involving the third type of clopen decreasing sets. Let $\{y\} \in \mathcal{F}_{I}$. Then $\{y\} \in \mathcal{F}$ and $y \in I$. Suppose $x \neq y$. Notice that $\{x, y\} \in \mathcal{F}$. We have $(\zeta(x)] \cup(\zeta(y)]=\zeta(\{x, y\}) \cup S$ and $(\zeta(x)] \cap(\zeta(y)]=S \backslash\{x, y\}$.

Now we show that $K(\mathcal{F})$ is closed under pseudocomplementation. Let $X \in \mathcal{F}$. If $X=\emptyset$, then $X^{*}=S \cup \zeta(S)$. If $X=\{x\}$ with $x \in I$, then $\{x\} \in \mathcal{F}_{I}$ and $X^{*}=(S \cup \zeta(S)) \backslash[x)=$ $\{\zeta(x)\} \cup(S \backslash\{x\})=(\zeta(x)]$. For the remaining cases, $X^{*}=(S \cup \zeta(S)) \backslash[X)=(S \cup \zeta(S)) \backslash(X \cup \zeta(S))=$ $S \backslash X$. Further, we have $(\zeta(X) \cup S)^{*}=\emptyset$. It remains to show that $K(\mathcal{F})$ is closed for the third type of clopen decreasing set. Let $\{x\} \in \mathcal{F}_{I}$. Then $\{x\} \in \mathcal{F}$ and $x \in I$ and we have $(\zeta(x)]^{*}=(S \cup \zeta(S)) \backslash[\{\zeta(x)\} \cup(S \backslash\{x\}))=\{x\}$.

Finally we show that $K(\mathcal{F})$ is closed for the de Morgan operation. Let $X \in \mathcal{F}$. We have $X^{\prime}=(S \cup \zeta(S)) \backslash \zeta(X)=\zeta(S \backslash X) \cup S$ and $(\zeta(X) \cup S)^{\prime}=(S \cup \zeta(S)) \backslash(X \cup \zeta(S))=S \backslash X$. If $\{x\} \in \mathcal{F}_{I}$, we have $(\zeta(x)]^{\prime}=(S \cup \zeta(S)) \backslash(\{x\} \cup(\zeta(S) \backslash\{\zeta(x)\}))=\{\zeta(x)\} \cup(S \backslash\{x\})=(\zeta(x)]$, thereby completing the justification of the claim.

Let $N$ be a positive integer. Let $\left\{X_{i}: 0 \leq i<N\right\}$ be a set of clopen decreasing sets of $Q_{6}(I, S)$. For each $i, 0 \leq i<N, X_{i}=\left(X_{i} \cap S\right) \cup\left(X_{i} \cap \zeta(S)\right)=\left(X_{i} \cap S\right) \cup \zeta\left(\zeta\left(X_{i}\right) \cap S\right)$ and $X_{i} \cap S$ and $\zeta\left(X_{i}\right) \cap S$ are clopen sets of $S$. Consider $T=\left\{X_{i} \cap S: 0 \leq i<N\right\} \cup\left\{\zeta\left(X_{i}\right) \cap S: 0 \leq i<N\right\}$. This set has, at most, $N_{1}=2 N$ elements. Let $\mathcal{B}$ be the Boolean algebra of clopen sets of $S$ and $\mathcal{F}$ its Boolean subalgebra generated by $T$. Since a free Boolean algebra with $N_{1}$ generators has $2^{\left(2^{N_{1}}\right)}$ elements, $\mathcal{F}$ has at most $N_{2}=2^{\left(2^{N_{1}}\right)}$ elements. By the above claim, $K(\mathcal{F})$ is a subalgebra of $E\left(Q_{6}(I, S)\right)$. It is obvious that $K(\mathcal{F})$ has, at most, $3 N_{2}$ elements. It remains to show that, for every $i, 0 \leq i<N$, $X_{i} \in K(\mathcal{F})$. We know that $X_{i}=\left(X_{i} \cap S\right) \cup \zeta\left(\zeta\left(X_{i}\right) \cap S\right)$. For each $i, X_{i} \cap S \in T \subseteq \mathcal{F}$ and $\zeta\left(X_{i}\right) \cap S \in T \subseteq \mathcal{F}$. If $\zeta\left(X_{i}\right) \cap S=\emptyset$, then $X_{i}=X_{i} \cap S \in \mathcal{F} \subseteq K(\mathcal{F})$. Suppose $\left|\zeta\left(X_{i}\right) \cap S\right| \geq 2$ or $\zeta\left(X_{i}\right) \cap S=\{x\}$ with $x \notin I$. Then $\left|\zeta\left(\zeta\left(X_{i}\right) \cap S\right)\right| \geq 2$ or $\zeta\left(\zeta\left(X_{i}\right) \cap S\right)=\{\zeta(x)\}$ with $x \notin I$. As $\zeta\left(\zeta\left(X_{i}\right) \cap S\right) \subseteq X_{i}$ and $X_{i}$ is decreasing, $S \subseteq X_{i}$ and, consequently, $X_{i}=S \cup \zeta\left(\zeta\left(X_{i}\right) \cap S\right) \in K(\mathcal{F})$. 
Finally, suppose $\zeta\left(X_{i}\right) \cap S=\{x\}$ with $x \in I$. Then $\zeta\left(\zeta\left(X_{i}\right) \cap S\right)=\{\zeta(x)\}$ and $X_{i}=\left(X_{i} \cap S\right) \cup\{\zeta(x)\}$ and, as $X_{i}$ is decreasing, $X_{i}=\left(X_{i} \cap S\right) \cup(\zeta(x)]$ with $\{x\} \in \mathcal{F}$ and $x \in I$. So, $X_{i} \in K(\mathcal{F})$.

Thus, the subalgebra of $E\left(Q_{6}(I, S)\right)$ generated by $\left\{X_{i}: 0 \leq i<N\right\}$ has at most $N^{\prime}=3 N_{2}=$ $3 \times 2^{\left(2^{N_{1}}\right)}=3 \times 2^{\left(2^{2 N}\right)}$ elements.

For any $i, 0 \leq i \leq 5$, and for any pair $(m, n)$ with $3 \leq n<\omega$ and $0 \leq m \leq n$, let

$$
L_{i}=E\left(Q_{i}\right) \quad \text { and } \quad L_{6}(m, n)=E\left(Q_{6}(m, n)\right) .
$$

In view of Theorem 4.10 the following corollary is immediate.

COROLLARY 4.11. The variety $\mathbf{M}_{1}$ is generated by its finite simple algebras, which are, up to isomorphism, $L_{i}, 0 \leq i \leq 5$, and $L_{6}(m, n), 3 \leq n<\omega, 0 \leq m \leq n$.

To show that the free algebra in $\mathbf{K}_{2}$ on one generator, $F_{\mathbf{K}_{2}}(1)$, is infinite we are going to consider the sequence of algebras of $\mathbf{K}_{2}$ presented in the following example.

EXAMPLE 4.12. For $5 \leq n<\omega$, let $S_{n}=\left\{x_{i}: 0 \leq i<n\right\}$ and $T_{n}=\left\{y_{i}: 0 \leq i<n\right\}$ be disjoint $n$-element sets. Define on $S_{n} \cup T_{n}$ the partial order $\leq$ induced by, for $0 \leq i, j<n$,

$$
x_{i}<y_{j} \quad \text { iff } \quad i \notin\{j-1, j+1\} .
$$

Let $\zeta: S_{n} \cup T_{n} \rightarrow S_{n} \cup T_{n}$ be defined by $\zeta\left(x_{i}\right)=y_{i}$ and $\zeta\left(y_{i}\right)=x_{i}$, for any $0 \leq i<n$. Consider $P_{n}=\left(S_{n} \cup T_{n} ; \leq, \zeta\right)$ which is a $p m$-space and its dual $p m$-algebra $K_{n}=E\left(P_{n}\right)$.

As $\operatorname{Min}\left(P_{n}\right)=S_{n}$ and $\operatorname{Max}\left(P_{n}\right)=T_{n}$, we have that $P_{n}=\operatorname{Min}\left(P_{n}\right) \cup \operatorname{Max}\left(P_{n}\right)$ and then $K_{n}$ is a regular pm-algebra, by Theorem 3.1. Moreover, since $\zeta\left(y_{i}\right)=x_{i}<y_{i}=\zeta\left(x_{i}\right)$ for every $0 \leq i<n$, $K_{n}$ is a regular pseudocomplemented Kleene algebra.

The algebra $K_{n}$ is of range 2 , that is $K_{n} \in \mathbf{K}_{2}$. In fact, since $\ell_{\zeta}(x, y)=\ell_{\zeta}(\zeta(x), \zeta(y))=$ $\ell_{\zeta}(x, \zeta(y))=\ell_{\zeta}(\zeta(x), y)$, for any $x, y \in P_{n}$, it suffices to show that, for $0 \leq i, j<n, \ell_{\zeta}\left(y_{i}, y_{j}\right) \leq 2$. Let $0 \leq i, j<n$, choose $0 \leq k<n$ such $k \notin\{i-1, i+1, j-1, j+1\}$. Then $x_{k}<y_{i}, y_{j}$ and $\ell\left(y_{i}, y_{j}\right) \leq 2$. So $\ell_{\zeta}\left(y_{i}, y_{j}\right) \leq 2$. Thus $P_{n}$ is of $\zeta$-width 2 and then $K_{n}$ is of range 2 , by Corollary 3.7 .

Notice that $K_{n} \notin \mathbf{K}_{1}$, since $\ell\left(y_{0}, y_{1}\right)=2$ and $\ell\left(y_{0}, \zeta\left(y_{1}\right)\right)=\ell\left(y_{0}, x_{1}\right)=3$, so $\ell_{\zeta}\left(y_{0}, y_{1}\right)=2$.

THEOREM 4.13. The variety $\mathbf{K}_{2}$ is not locally finite, in fact the free algebra on one generator $F_{\mathbf{K}_{2}}(1)$ is infinite.

Proof. We show that, for each $5 \leq n<\omega$, there exists a subalgebra $A_{n}$ of $K_{n}$ that is generated by one element and has cardinality, at least, $n$.

Let $5 \leq n<\omega$. First notice that all the sets $\left\{x_{i}\right\}$ are decreasing. We have $\left[x_{0}\right)=\left\{x_{0}\right\} \cup\left(T_{n} \backslash\right.$ $\left.\left\{y_{1}\right\}\right),\left\{x_{0}\right\}^{*}=P_{n} \backslash\left[x_{0}\right)=\left\{y_{1}\right\} \cup\left(S_{n} \backslash\left\{x_{0}\right\}\right)$ and $\left\{x_{0}\right\}^{* \prime}=\zeta\left(P_{n} \backslash\left\{x_{0}\right\}^{*}\right)=\zeta\left(P_{n} \backslash\left(P_{n} \backslash\left[x_{0}\right)\right)\right)=$ $\zeta\left(\left[x_{0}\right)\right)=\left\{y_{0}\right\} \cup\left(S_{n} \backslash\left\{x_{1}\right\}\right)$. Moreover, for $1 \leq i<n-1$, we have $\left[x_{i}\right)=\left\{x_{i}\right\} \cup\left(T_{n} \backslash\left\{y_{i-1}, y_{i+1}\right\}\right)$, $\left\{x_{i}\right\}^{*}=\left\{y_{i-1}, y_{i+1}\right\} \cup\left(S_{n} \backslash\left\{x_{i}\right\}\right)$ and $\left\{x_{i}\right\}^{* \prime}=\zeta\left(\left[x_{i}\right)\right)=\left\{y_{i}\right\} \cup\left(S_{n} \backslash\left\{x_{i-1}, x_{i+1}\right\}\right)$.

Consider $X=\left\{x_{0}\right\}$ and let $\langle X\rangle$ denote the subalgebra of $K_{n}$ generated by $X$. We will show that, for every $0 \leq i<n,\left\{x_{i}\right\} \in\langle X\rangle$. Since $X^{* \prime}=\left\{x_{0}\right\}^{* \prime}=\left\{y_{0}\right\} \cup\left(S_{n} \backslash\left\{x_{1}\right\}\right)$, we have that $X^{* * *}=P_{n} \backslash\left[X^{* \prime}\right)=\left\{x_{1}\right\} \in\langle X\rangle$. Proceeding inductively, let $1 \leq i<n-1$ and suppose that $\left\{x_{k}\right\} \in\langle X\rangle$, for any $0 \leq k \leq i$. Since $\left\{x_{i}\right\}^{* \prime}=\left\{y_{i}\right\} \cup\left(S_{n} \backslash\left\{x_{i-1}, x_{i+1}\right\}\right)$, we have that $\left\{x_{i}\right\}^{* * *}=P_{n} \backslash\left[\left\{x_{i}\right\}^{* \prime}\right)=\left\{x_{i-1}, x_{i+1}\right\}$. As $x_{i-1} \notin\left\{x_{i-1}\right\}^{*}$ but $x_{i+1} \in\left\{x_{i-1}\right\}^{*}$, we have 
$\left\{x_{i}\right\}^{* * *} \cap\left\{x_{i-1}\right\}^{*}=\left\{x_{i+1}\right\}$ and, consequently, $\left\{x_{i+1}\right\} \in\langle X\rangle$, since both $\left\{x_{i}\right\}^{* *},\left\{x_{i-1}\right\}^{*} \in\langle X\rangle$.

As all the regular pseudocomplemented Kleene algebras $A_{n}$ are homomorphic images of $F_{\mathbf{K}_{2}}(1)$, this algebra must be infinite.

COROLLARY 4.14. For $2 \leq n<\omega$, the varieties $\mathbf{K}_{n}$ and $\mathbf{M}_{n}$ are not locally finite.

Proof. It is immediate from Theorem 4.13 that $F_{\mathbf{V}}(1)$ is infinite, where $\mathbf{V}$ is any of the mentioned varieties.

\section{$5 \quad$ Lattices of subvarieties of $\mathbf{K}_{1}$ and $\mathbf{M}_{1}$}

As observed in Section 1 the following theorem follows from [22].

THEOREM 5.1. (22])

(1) $L_{V}\left(\mathbf{K}_{0}\right)$ is isomorphic to a 3-element chain,

(2) $L_{V}\left(\mathbf{M}_{0}\right)$ is isomorphic to the 5-element lattice $\mathbf{1} \oplus(\mathbf{2} \times \mathbf{2})$.

Now we focus on $L_{V}\left(\mathbf{K}_{1}\right)$ and on $L_{V}\left(\mathbf{M}_{1}\right)$.

As the variety of pseudocomplemented de Morgan algebras is congruence-distributive, it is well known (cf. [10]) that its lattice of subvarieties is distributive.

Let $\mathcal{K}$ be a class of algebras of the same similarity type. As usual, we denote by $\mathbf{H}(\mathcal{K}), \mathbf{S}(\mathcal{K})$, $\mathbf{I}(\mathcal{K})$, and $\mathbf{V}(\mathcal{K})$ respectively, the class of all homomorphic images of members of $\mathcal{K}$, the class of all subalgebras of members of $\mathcal{K}$, the class of all isomorphic images of members of $\mathcal{K}$, and the variety generated by $\mathcal{K}$. If $\mathcal{K}$ has finitely many members, say $\mathcal{K}=\left\{K_{0}, \ldots, K_{n}\right\}$ with $n<\omega$, the mentioned classes will be denoted by $\mathbf{H}\left(K_{0}, \ldots, K_{n}\right), \mathbf{S}\left(K_{0}, \ldots, K_{n}\right), \mathbf{I}\left(K_{0}, \ldots, K_{n}\right)$ and $\mathbf{V}\left(K_{0}, \ldots, K_{n}\right)$, respectively.

THEOREM 5.2. Let $n<\omega$ and let $\mathcal{K}$ be a class of simple algebras of $\mathbf{M}_{n}$ such that $\mathbf{V}(\mathcal{K})$ is locally finite. Then every finite simple algebra in $\mathbf{V}(\mathcal{K})$ belongs to $\mathbf{I S}(\mathcal{K})$.

Proof. We have already observed that any subalgebra of a simple algebra in $\mathbf{M}_{n}$ is also simple. So $\operatorname{HS}(\mathcal{K})=\mathbf{I S}(\mathcal{K})$. Now the conclusion follows immediately from [15, Theorem 4.104].

The set $S i_{F}=\left\{L_{i}: 0 \leq i \leq 5\right\} \cup\left\{L_{6}(m, n): 3 \leq n<\omega\right.$ and $\left.0 \leq m \leq n\right\}$ consists of precisely one algebra from each of the isomorphism classes of the finite simple algebras in $\mathbf{M}_{1}$. Since, by Theorem 4.10, $\mathbf{M}_{1}$ is locally finite, any of its subvarieties is generated by its finite simple members. So any subvariety of $\mathbf{M}_{1}$ is generated by some subset $\mathcal{K}$ of $S i_{F}$. Moreover, in view of the last theorem, every finite simple member of $\mathbf{V}(\mathcal{K})$ lies in $\mathbf{I S}(\mathcal{K})$.

As observed in Section 2 if $P$ and $Q$ are the dual spaces of the $p m$-algebras $L$ and $K$, respectively, then $L$ is isomorphic to a subalgebra of $K$ if and only if there exists a surjective $p m$-morphism from $Q$ onto $P$. If $L$ and $K$ are finite, the topology in $P$ and $Q$ is the discrete one and plays no role. Notice that for any $p m$-morphism $\varphi: Q \rightarrow P$, we have $\varphi(\operatorname{Min}(Q)) \subseteq \operatorname{Min}(P)$ and $\varphi(\operatorname{Max}(Q)) \subseteq \operatorname{Max}(P)$ and, since $\varphi$ is order-preserving, if each $Q$ and $P$ is the disjoint union of the set of its minimal elements and the set of its maximal ones, we have $\varphi(x)<\varphi(y)$ whenever $x<y$. Moreover, for any surjective $p m$-morphism $\varphi: Q \rightarrow P$, we have $\varphi(\operatorname{Min}(Q))=\operatorname{Min}(P)$ and $\varphi(\operatorname{Max}(Q))=\operatorname{Max}(P)$. 
Ultimately to describe $L_{V}\left(\mathbf{M}_{1}\right)$ it will be necessary to determine precisely when $L_{6}(p, q)$ is a subalgebra of $L_{6}(m, n)$ for all $m, n, p, q$ with $3 \leq n<\omega, 0 \leq m \leq n, 3 \leq q<\omega$ and $0 \leq p \leq q$. The next two lemmas 5.3 and 5.5 pave the way for this, namely Theorem 5.6 .

LEMMA 5.3. Let $Q_{6}(I, S)$ and $Q_{6}(J, T)$ be finite. A map $\varphi: Q_{6}(I, S) \rightarrow Q_{6}(J, T)$ is a surjective pm-morphism if and only if the following conditions are satisfied

(1) $\varphi(S)=T$ and $\varphi(\zeta(x))=\zeta(\varphi(x))$, for any $x \in S$.

(2) $\varphi^{-1}(J) \subseteq I$.

(3) $\varphi(x) \neq \varphi(y)$, for distinct $x, y \in \varphi^{-1}(J)$.

(4) If $x \in I \backslash \varphi^{-1}(J)$, then there exists $u \in S \backslash \varphi^{-1}(J)$ such that $u \neq x$ and $\varphi(u)=\varphi(x)$.

Proof. We have that $\operatorname{Min}\left(Q_{6}(I, S)\right)=S, \operatorname{Max}\left(Q_{6}(I, S)\right)=\zeta(S)$, whilst $\operatorname{Min}\left(Q_{6}(J, T)\right)=T$ and $\operatorname{Max}\left(Q_{6}(J, T)\right)=\zeta(T)$. Moreover, $S$ and $\zeta(S)$ are disjoint and so are $T$ and $\zeta(T)$.

Suppose $\varphi: Q_{6}(I, S) \rightarrow Q_{6}(J, T)$ is a surjective $p m$-morphism.

(1) It is obvious since $\varphi(S)=\varphi\left(\operatorname{Min}\left(Q_{6}(I, S)\right)\right)=\operatorname{Min}\left(Q_{6}(J, T)\right)=T$ and $\varphi \circ \zeta=\zeta \circ \varphi$.

(2) Let $x \in \varphi^{-1}(J)$. Then $\varphi(x) \in J$ and, consequently, $x \in S$ and $\varphi(x) \nless \zeta(\varphi(x))=\varphi(\zeta(x))$. If $x \notin I$, then $x<\zeta(x)$ and, consequently, $\varphi(x)<\varphi(\zeta(x))$, a contradiction. Thus, $x \in I$.

(3) Let $x, y \in \varphi^{-1}(J)$ be such that $x \neq y$. Then $x<\zeta(y)$ and, consequently, $\varphi(x)<\varphi(\zeta(y))=$ $\zeta(\varphi(y))$. If $\varphi(x)=\varphi(y)$, we would have $\varphi(x)<\zeta(\varphi(x))$, a contradiction, since $\varphi(x) \in J$. Thus, $\varphi(x) \neq \varphi(y)$.

(4) Suppose that $x \in I \backslash \varphi^{-1}(J)$. That is, $x \in I$ and $\varphi(x) \notin J$. Then $x \nless \zeta(x)$ and $\varphi(x)<\zeta(\varphi(x))=\varphi(\zeta(x))$. So, $\varphi(x) \in \operatorname{Min}(\varphi(\zeta(x)))=\varphi(\operatorname{Min}(\zeta(x)))$ and, consequently, there exists $u \in \operatorname{Min}(\zeta(x))$ such that $\varphi(x)=\varphi(u)$. It is obvious that $u \in S$ and, as $\varphi(u)=\varphi(x) \notin J$, we have that $u \in S \backslash \varphi^{-1}(J)$. From $u<\zeta(x)$ and $x \nless \zeta(x)$, it follows that $u \neq x$.

Conversely, suppose that $\varphi$ satisfies (1) - (4). By (1), $\varphi$ is onto and $\varphi \circ \zeta=\zeta \circ \varphi$. To prove that $\varphi$ is order-preserving, consider $x, y \in S$ such that $x<\zeta(y)$. If $\varphi(x) \nless \varphi(\zeta(y))=\zeta(\varphi(y))$, then $\varphi(x)=\varphi(y) \in J$ and, applying (2) and (3), $x=y \in \varphi^{-1}(J) \subseteq I$, a contradiction, since $x<\zeta(y)$. Finally, let $x \in S$. As $S=\operatorname{Min}\left(Q_{6}(I, S)\right), T=\operatorname{Min}\left(Q_{6}(J, T)\right)$ and, by $(1), \varphi(S)=T$, we have that $\operatorname{Min}(\varphi(x)) \subseteq \varphi(\operatorname{Min}(x))$ trivially. To prove that $\operatorname{Min}(\varphi(\zeta(x))) \subseteq \varphi(\operatorname{Min}(\zeta(x)))$, take $z \in \operatorname{Min}(\varphi(\zeta(x)))$. Then $z \in T=\varphi(S)$ and $z<\varphi(\zeta(x))=\zeta(\varphi(x))$. Let $y \in S$ be such that $z=\varphi(y)$ and suppose that $y \nless \zeta(x)$. Then $y=x \in I$ and $\varphi(x)=z<\zeta(\varphi(x))$. So $x \notin \varphi^{-1}(J)$. Applying (4), there exists $u \in S \backslash \varphi^{-1}(J)$ such that $u \neq x$ and $\varphi(u)=\varphi(x)=z$. Since $u \neq x$, we have $u<\zeta(x)$ and then $z \in \varphi(\operatorname{Min}(\zeta(x)))$. So, $\operatorname{Min}(\varphi(\zeta(x))) \subseteq \varphi(\operatorname{Min}(\zeta(x)))$. Thus, $\varphi$ is a surjective $p m$-morphism.

It is straightforward to conclude that for $3 \leq k \leq n<\omega$ and $0 \leq m \leq k$, there is a surjective $p m$-morphism from $Q_{6}(m, n)$ onto $Q_{6}(m, k)$. Simply apply the previous lemma to any $\varphi: Q_{6}(I, S) \rightarrow Q_{6}(J, T)$ where $|I|=|J|$ and $|S \backslash I| \geq|T \backslash J|$ and $\varphi_{\left.\right|_{I}}: I \rightarrow J$ is a bijection and $\varphi_{\mid(S \backslash I)}: S \backslash I \rightarrow T \backslash J$ is an onto mapping.

Now we introduce some notation. For each $i, 0 \leq i \leq 5$, and each pair $(m, n)$ such that $3 \leq n<\omega$ and $0 \leq m \leq n$, let

$$
\mathbf{V}_{i}=\mathbf{V}\left(L_{i}\right) \text { and } \mathbf{V}_{m, n}=\mathbf{V}\left(L_{6}(m, n)\right) .
$$

Also let 


$$
\mathbf{V}_{\omega, \omega}=\mathbf{V}\left(\left\{L_{6}(m, n): 3 \leq n<\omega \text { and } 0 \leq m<n\right\}\right),
$$

and, for each $m<\omega$,

$$
\mathbf{V}_{m, \omega}=\mathbf{V}\left(\left\{L_{6}(m, n): 3 \leq n<\omega \text { and } m<n\right\}\right) .
$$

THEOREM 5.4. $L_{V}\left(\mathbf{K}_{\mathbf{1}}\right)$ is isomorphic to an $\omega+1$ chain.

Proof. Analysing the algebras of $S i_{F}$, we conclude that only $L_{0}, L_{2}, L_{5}$ and $L_{6}(0, n)$, for any $3 \leq n<\omega$, are in $\mathbf{K}_{1}$. As we have just observed, for $3 \leq n<m$, there is a surjective $p m$-morphism from $Q_{6}(0, m)$ onto $Q_{6}(0, n)$, but, obviously, there is no surjective map from $Q_{6}(0, n)$ onto $Q_{6}(0, m)$. So, $L_{6}(0, n) \in \mathbf{I S}\left(L_{6}(0, m)\right)$, but $L_{6}(0, m) \notin \mathbf{I S}\left(L_{6}(0, n)\right)$ and, consequently, $\mathbf{V}_{0, n}<\mathbf{V}_{0, m}$. It is also clear that there are surjective $p m$-morphisms from $Q_{6}(0,3)$ onto $Q_{5}$, from $Q_{5}$ onto $Q_{2}$, and from $Q_{2}$ onto $Q_{0}$, but not the other way around. That is, $L_{V}\left(\mathbf{K}_{\mathbf{1}}\right)$ is isomorphic to the $\omega+1$ chain

$$
\mathbf{T}<\mathbf{V}_{0}<\mathbf{V}_{2}<\mathbf{V}_{5}<\mathbf{V}_{0,3}<\ldots<\mathbf{V}_{0, n}<\ldots<\mathbf{K}_{\mathbf{1}},
$$

where $\mathbf{T}$ is the trivial subvariety.

Observe that, in terms of the preceding notation, $\mathbf{K}_{\mathbf{1}}=\mathbf{V}_{0, \omega}$.

The lattice $L_{V}\left(\mathbf{M}_{\mathbf{1}}\right)$ is a little more complex. Our first goal is to analyse when $L_{6}(p, q) \in \mathbf{V}_{m, n}$, that is, when $L_{6}(p, q) \in \mathbf{I S}\left(L_{6}(m, n)\right)$.

LEMMA 5.5. Let $Q_{6}(I, S)$ and $Q_{6}(J, T)$ be finite and $\varphi: Q_{6}(I, S) \rightarrow Q_{6}(J, T)$ be a surjective pm-morphism. Let $x, y, u, v$ be distinct elements of $S$ such that $x, y \in I \backslash \varphi^{-1}(J), u, v \in S \backslash \varphi^{-1}(J)$ and $\varphi(u)=\varphi(x) \neq \varphi(y)=\varphi(v)$. If, for any finite set $T_{1}$ that properly contains $T$, there is no surjective pm-morphism from $Q_{6}(I, S)$ onto $Q_{6}\left(J, T_{1}\right)$, then $u \in I \backslash \varphi^{-1}(J)$ or $v \in I \backslash \varphi^{-1}(J)$.

Proof. Suppose that for any finite set $T_{1}$ that properly contains $T$, there is no surjective pmmorphism from $Q_{6}(I, S)$ onto $Q_{6}\left(J, T_{1}\right)$. As $\varphi$ is a surjective $p m$-morphism, it satisfies conditions (1)-(4) of Lemma 5.3. Notice that $\varphi(x), \varphi(y) \notin J$, since $x, y \in I \backslash \varphi^{-1}(J)$. As $\varphi(u)=\varphi(x)$ and $\varphi(v)=\varphi(y)$, we have that $u, v \notin \varphi^{-1}(J)$. Thus it only remains to prove that $u \in I$ or $v \in I$. Suppose that $u \notin I$ and $v \notin I$. Choose some completely new element $w \notin T \cup \zeta(T)$. Consider $Q_{6}\left(J, T_{1}\right)$ where $T_{1}=T \cup\{w\}$. Define $\psi: Q_{6}(I, S) \rightarrow Q_{6}\left(J, T_{1}\right)$ by, for $z \in S$,

$$
\begin{aligned}
& \psi(z)= \begin{cases}\varphi(z) & \text { if } \varphi(z) \notin\{\varphi(x), \varphi(y)\} \\
\varphi(x) & \text { if } \varphi(z) \in\{\varphi(x), \varphi(y)\} \text { and } z \notin\{u, v\} \\
\varphi(y) & \text { if } z=u \\
w & \text { if } z=v\end{cases} \\
& \psi(\zeta(z))=\zeta(\psi(z))
\end{aligned}
$$

and

We will prove that $\psi$ satisfies (1)-(4) of Lemma 5.3, obtaining a contradiction.

(1) We have that $\psi(v)=w, \psi(u)=\varphi(y)$ and $\psi(x)=\varphi(x)$. Let $b \in T_{1} \backslash\{w, \varphi(y), \varphi(x)\}$. Then $b \in T \backslash\{\varphi(y), \varphi(x)\}$ and, since $\varphi(S)=T$, there exists $z \in S$ such that $\varphi(z)=b \notin\{\varphi(y), \varphi(x)\}$ and we have $\psi(z)=\varphi(z)=b$. So $\psi(S)=T_{1}$. By definition, $\psi(\zeta(z))=\zeta(\psi(z))$ for any $z \in S$.

(2) Although $\psi^{-1}(J) \subseteq \varphi^{-1}(J)$ is sufficient to conclude (2), we will show that $\psi^{-1}(J)=\varphi^{-1}(J)$ and that $\psi(z)=\varphi(z)$, for $z \in \psi^{-1}(J)$, which will be used to prove (3) and (4). If $z \in \varphi^{-1}(J)$, then $\varphi(z) \in J$ and so $\varphi(z) \notin\{\varphi(x), \varphi(y)\}$. Thus, $\psi(z)=\varphi(z) \in J$ and $z \in \psi^{-1}(J)$. Conversely, let $z \in \psi^{-1}(J)$. Then $\psi(z) \in J$. If $\varphi(z) \in\{\varphi(x), \varphi(y)\}$, we would have $\psi(z) \in\{w, \varphi(y), \varphi(x)\}$, 
a contradiction, since none of the elements of this set belongs to $J$. So $\varphi(z) \notin\{\varphi(x), \varphi(y)\}$ and, consequently, $\varphi(z)=\psi(z) \in J$. Thus $\psi^{-1}(J)=\varphi^{-1}(J)$. Consequently, $\psi^{-1}(J)=\varphi^{-1}(J) \subseteq I$.

(3) Let $z_{1}, z_{2} \in \psi^{-1}(J)$ be distinct. We just proved in (2) that $\psi^{-1}(J)=\varphi^{-1}(J)$. Also, from the proof of (2), we know that $\psi\left(z_{1}\right)=\varphi\left(z_{1}\right)$ and $\psi\left(z_{2}\right)=\varphi\left(z_{2}\right)$. As, by (3) of Lemma 5.3. $\varphi\left(z_{1}\right) \neq \varphi\left(z_{2}\right)$, we have $\psi\left(z_{1}\right) \neq \psi\left(z_{2}\right)$.

(4) Suppose that $z \in I \backslash \psi^{-1}(J)$. As $u \notin I$ and $v \notin I$ by hypothesis, we have $z \notin\{u, v\}$. We consider two cases. First, suppose $\varphi(z) \in\{\varphi(x), \varphi(y)\}$, then $\psi(z)=\varphi(x)=\psi(x)=\psi(y)$ by definition of $\psi$, and, since $x \neq y$, we have $z \neq x$ or $z \neq y$. Both $x, y \in I \backslash \varphi^{-1}(J)=I \backslash \psi^{-1}(J)$. Now suppose $\varphi(z) \notin\{\varphi(x), \varphi(y)\}$. Then $\psi(z)=\varphi(z)$ by definition of $\psi$. By the proof of (2), we know that $\psi^{-1}(J)=\varphi^{-1}(J)$ and, since $z \in I \backslash \psi^{-1}(J)$, we have $z \in I \backslash \varphi^{-1}(J)$. As $\varphi$ is a surjective pm-morphism, applying (4) of Lemma [5.3, there exists $z_{0} \in S \backslash \varphi^{-1}(J)=S \backslash \psi^{-1}(J)$ such that $z_{0} \neq z$ and $\varphi\left(z_{0}\right)=\varphi(z)$. So $\varphi\left(z_{0}\right) \notin\{\varphi(x), \varphi(y)\}$ and we have $\psi\left(z_{0}\right)=\varphi\left(z_{0}\right)=\varphi(z)=\psi(z)$.

Recall that, for a real number $r$, the largest integer that does not exceed $r$ is called the floor of $r$ and denoted $\lfloor r\rfloor$.

We are now ready to determine when $L_{6}(p, q) \in \mathbf{V}_{m, n}$ and, as can be seen, the answer is rather curious.

THEOREM 5.6. Let $3 \leq n, q<\omega, 0 \leq m \leq n$ and $0 \leq p \leq q$. Then the following are equivalent:

(1) $L_{6}(p, q) \in \mathbf{V}_{m, n}$,

(2) $p \leq m$, and

either

(a) $p=q=m=n$,

or

(b) $p<q$ and $q \leq p+\lfloor(m-p) / 2\rfloor+n-m$.

Proof. Say $Q_{6}(m, n)=Q_{6}(I, S)$ where $I \subseteq S,|I|=m$ and $|S|=n$, and $Q_{6}(p, q)=Q_{6}(J, T)$ where $J \subseteq T,|J|=p$ and $|T|=q$. We know that $L_{6}(p, q) \in \mathbf{V}_{m, n}$ if and only if there is a surjective $p m$-morphism from $Q_{6}(I, S)$ onto $Q_{6}(J, T)$. Suppose that (1) holds and let $\varphi$ be such a surjective pm-morphism. Then, by (1) of Lemma 5.3, $\varphi(S)=T$ and $\varphi(\zeta(S))=\zeta(T)$. It is obvious that $q \leq n$. Moreover, $\varphi\left(\varphi^{-1}(J)\right)=J$ and, applying (3) of Lemma [5.3, we have $p=|J|=\left|\varphi^{-1}(J)\right|$. But $\varphi^{-1}(J) \subseteq I$, by (2) of the same lemma, and then $p=\left|\varphi^{-1}(J)\right| \leq|I|=m$. So it is always the case that $p \leq m$. Now we have 2 cases to consider.

Case 1: $p=q$. Then $J=T$ and so, $\varphi^{-1}(J)=\varphi^{-1}(T)=S$. Thus $p=n$ and, since $p \leq m \leq n$, we conclude that $p=m=n$. So $p=q=m=n$, as required.

Case 2: $p<q$. To show that $q \leq p+\lfloor(m-p) / 2\rfloor+n-m$, we may consider that $q$ is the greatest integer for which there exists a surjective $p m$-morphism from $Q_{6}(m, n)$ onto $Q_{6}(p, q)$. As $\varphi(S)=T$, we have $\varphi\left(S \backslash \varphi^{-1}(J)\right)=T \backslash J$. Since $p=\left|\varphi^{-1}(J)\right|$ and $\varphi^{-1}(J) \subseteq I$, we have $\left|I \backslash \varphi^{-1}(J)\right|=m-p$.

If $m-p=0$, then $\lfloor(m-p) / 2\rfloor=0$ and $p+\lfloor(m-p) / 2\rfloor+n-m=p+0+n-p=n$ and we have already observed that $q \leq n$.

Suppose $m-p=1$, that is $\left|I \backslash \varphi^{-1}(J)\right|=1$. Then $I \backslash \varphi^{-1}(J)$ is a singleton, say $I \backslash \varphi^{-1}(J)=\{x\}$. By (4) of Lemma [5.3. there exists $u \in S \backslash \varphi^{-1}(J)$ such that $u \neq x$ and $\varphi(u)=\varphi(x)$. Thus, $q=|J|+|T \backslash J|=p+\left|\varphi\left(S \backslash \varphi^{-1}(J)\right)\right| \leq p+\left(\left|S \backslash \varphi^{-1}(J)\right|-1\right)=p+n-p-1=n-1=$ 
$p+\lfloor(m-p) / 2\rfloor+n-m$.

Finally, suppose that $m-p \geq 2$, that is $\left|I \backslash \varphi^{-1}(J)\right| \geq 2$. As $\varphi^{-1}(J) \subseteq I$, we have $T \backslash J=\varphi\left(S \backslash \varphi^{-1}(J)\right)=\varphi\left(\left(I \backslash \varphi^{-1}(J)\right) \cup(S \backslash I)\right)$. Notice that $|S \backslash I|=n-m$. Consider $\theta$ the equivalence relation on $I \backslash \varphi^{-1}(J)$ defined by $(x, y) \in \theta$ iff $\varphi(x)=\varphi(y)$. Suppose that there are $k$ equivalence classes. Then $|T \backslash J| \leq k+n-m$. If each class has at least 2 elements then $m-p=\left|I \backslash \varphi^{-1}(J)\right| \geq 2 k$, implying $(m-p) / 2 \geq k$. So $\lfloor(m-p) / 2\rfloor \geq k$. Thus, $q=|J|+|T \backslash J| \leq p+k+n-m \leq p+\lfloor(m-p) / 2\rfloor+n-m$, as required. Now suppose that there exists a class with cardinality 1 . Then, as $\left|I \backslash \varphi^{-1}(J)\right|=m-p \geq 2$, we have that $k \geq 2$. Let $a_{1} / \theta, a_{2} / \theta, \ldots, a_{k} / \theta$ be the equivalence classes with $a_{1} / \theta=\left\{a_{1}\right\}$. For each $i, 2 \leq i \leq k$, we have $a_{1}, a_{i} \in I \backslash \varphi^{-1}(J)$ and $\varphi\left(a_{1}\right) \neq \varphi\left(a_{i}\right)$. By (4) of Lemma 5.3 there exists $u \in S \backslash \varphi^{-1}(J)$ such that $u \neq a_{1}$ and $\varphi(u)=\varphi\left(a_{1}\right)$ and there exists $v_{i} \in S \backslash \varphi^{-1}(J)$ such that $v_{i} \neq a_{i}$ and $\varphi\left(v_{i}\right)=\varphi\left(a_{i}\right)$. It is obvious that the elements $u, a_{1}, a_{i}, v_{i}$ are distinct and that $u \notin I$, as $a_{1} / \theta=\left\{a_{1}\right\}$. By the maximality of $q$ and applying Lemma 5.5 we conclude that $v_{i} \in I \backslash \varphi^{-1}(J)$ and consequently, $v_{i} \in a_{i} / \theta$. So, all the classes except $a_{1} / \theta$ have at least 2 elements and $m-p=\left|I \backslash \varphi^{-1}(J)\right| \geq 2(k-1)+1=2 k-1$ and $(m-p) / 2 \geq k-1 / 2$, so $\lfloor(m-p) / 2\rfloor \geq k-1$. Taking into account that $u \in S \backslash I$ and $\varphi(u)=\varphi\left(a_{1}\right)$, we have $q-p=|T \backslash J|=\left|\varphi\left(\left(I \backslash \varphi^{-1}(J)\right) \cup(S \backslash I)\right)\right| \leq k+(n-m-1)=(k-1)+(n-m) \leq\lfloor(m-p) / 2\rfloor+n-m$ and hence (2) follows.

Now, suppose (2) is true. We wish to prove that there is a surjective $p m$-morphism from $Q_{6}(I, S)$ onto $Q_{6}(J, T)$. If $p=q=m=n$, it is immediate. Now suppose that $p \leq m$ and $p<q \leq p+\lfloor(m-p) / 2\rfloor+n-m$. As for any $t \geq q$, there is a surjective $p m$-morphism from $Q_{6}(p, t)$ onto $Q_{6}(p, q)$, it is sufficient to prove the statement for $q=p+\lfloor(m-p) / 2\rfloor+n-m$.

As $p \leq m=|I|$ consider $M \subseteq I$ such that $|M|=p=|J|$ and let $\alpha$ be a bijection from $M$ onto $J$. Then $|I \backslash M|=m-p=2 k$ or $|I \backslash M|=m-p=2 k+1$, for some $k \geq 0$, and, in both cases, $\lfloor(m-p) / 2\rfloor=k$.

Suppose $k \geq 1$. Let $I \backslash M=\left\{a_{1}, \ldots, a_{k}\right\} \cup\left\{b_{1}, \ldots, b_{k}\right\} \cup B$, where $B=\emptyset$ if $|I \backslash M|=2 k$, and $B=\{b\}$ if $|I \backslash M|=2 k+1$. As $|T|=q=p+k+n-m$ and $|J|=p,|T \backslash J|=k+n-m \geq k$. Choose $k$ (distinct) elements in $T \backslash J$, say $c_{1}, \ldots, c_{k}$, and let $C=\left\{c_{1}, \ldots, c_{k}\right\}$. Let $\beta: I \backslash M \rightarrow C$ be defined by $\beta\left(a_{i}\right)=\beta\left(b_{i}\right)=c_{i}$, for any $1 \leq i \leq k$, and $\beta(b)=c_{k}$ if $B \neq \emptyset$. As the sets $T \backslash(J \cup C)$ and $S \backslash I$ both have cardinality $n-m$, consider a bijection $\gamma$ from $S \backslash I$ onto $T \backslash(J \cup C)$. Now let $\varphi: Q_{6}(I, S) \rightarrow Q_{6}(J, T)$ be defined by, for $x \in S$,

$$
\varphi(x)=\left\{\begin{array}{l}
\alpha(x) \text { if } x \in M \\
\beta(x) \text { if } x \in I \backslash M \\
\gamma(x) \text { if } x \in S \backslash I,
\end{array}\right.
$$

and

$$
\varphi(\zeta(x))=\zeta(\varphi(x)) .
$$

By Lemma 5.3, $\varphi$ is a surjective $p m$-morphism.

Now suppose $k=0$, that is $m-p=0$ or $m-p=1$ and, consequently, $I=M$ or $I \backslash M$ is a singleton, say $I \backslash M=\{b\}$. As $\lfloor(m-p) / 2\rfloor=0$, we have that $p+1 \leq q=p+n-m$. Then $n-m \geq 1$ and $|T \backslash J|=q-p=n-m=|S \backslash I|$. Choose $c \in T \backslash J$ and let $\beta$ be a bijection from $S \backslash I$ onto $T \backslash J$. Consider the map $\varphi: Q_{6}(I, S) \rightarrow Q_{6}(J, T)$ defined by, for $x \in S$, 


$$
\varphi(x)= \begin{cases}\alpha(x) & \text { if } x \in M \\ c & \text { if } x \in I \backslash M \\ \beta(x) & \text { if } x \in S \backslash I,\end{cases}
$$

and

$$
\varphi(\zeta(x))=\zeta(\varphi(x)) .
$$

By Lemma 5.3, $\varphi$ is a surjective $p m$-morphism.

COROLLARY 5.7. We have the following

(1) For $3 \leq p<\omega, 3 \leq n<\omega$ and $0 \leq m \leq n, L_{6}(p, p) \in \mathbf{I S}\left(L_{6}(m, n)\right)$ if and only if $p=m=n$.

(2) $\left|L_{V}\left(\mathbf{M}_{\mathbf{1}}\right)\right|=2^{\omega}$.

Proof. (1) Immediate from the previous theorem.

(2) Suppose $\mathcal{K}_{1}$ and $\mathcal{K}_{2}$ are distinct non-empty subsets of $\left\{L_{6}(n, n): 3 \leq n<\omega\right\}$. Without loss of generality, we may assume that there exists, say, $L_{6}(n, n) \in \mathcal{K}_{1} \backslash \mathcal{K}_{2}$ for some $3 \leq n<\omega$. If $L_{6}(n, n) \in \mathbf{V}\left(\mathcal{K}_{2}\right)$, then, by Theorem 5.2 $L_{6}(n, n) \in \mathbf{I S}\left(\mathcal{K}_{2}\right)$, that is $L_{6}(n, n) \in \mathbf{I S}\left(L_{6}(m, m)\right)$ for some $L_{6}(m, m) \in \mathcal{K}_{2}$, which is a contradiction, since $n \neq m$. So $L_{6}(n, n) \notin \mathbf{V}\left(\mathcal{K}_{2}\right)$ and $\mathbf{V}\left(\mathcal{K}_{1}\right) \neq \mathbf{V}\left(\mathcal{K}_{2}\right)$.

Although $L_{V}\left(\mathbf{M}_{\mathbf{1}}\right)$ is uncountable, with the aid of Theorem 5.6, its structure may still be understood.

Observe that $\mathbf{V}_{0} \subseteq \mathbf{V}$ for any non-trivial $\mathbf{V} \in L_{V}\left(\mathbf{M}_{\mathbf{1}}\right)$.

Further, since both $Q_{1}$ and $Q_{3}$ have an order component $Q$ for which $Q \cap \zeta(Q)=\emptyset$, we have that $L_{1}, L_{3} \notin \mathbf{V}(\mathcal{L})$ for $\mathcal{L} \subseteq S i_{F} \backslash\left\{L_{1}, L_{3}\right\}$.

First we consider the lattice of subvarieties of $\mathbf{L}=\mathbf{V}\left(L_{0}, L_{1}, L_{2}, L_{3}, L_{4}, L_{5}\right)$ which is a finite distributive lattice, whose non-zero join-irreducible elements are $\mathbf{V}_{i}, 0 \leq i \leq 5$. Inspection shows that the poset of these elements is

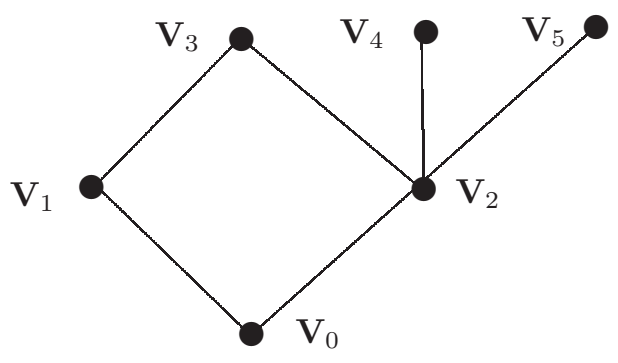

Figure 2

The lattice of subvarieties of $\mathbf{L}$ is isomorphic to the lattice of its decreasing sets. We conclude, in particular, that the variety $\mathbf{L}=\mathbf{V}\left(L_{0}, L_{1}, L_{2}, L_{3}, L_{4}, L_{5}\right)$ has 14 non-trivial subvarieties, namely: $\mathbf{V}_{i}, 0 \leq i \leq 5, \mathbf{V}_{1} \vee \mathbf{V}_{2}, \mathbf{V}_{1} \vee \mathbf{V}_{4}, \mathbf{V}_{1} \vee \mathbf{V}_{5}, \mathbf{V}_{3} \vee \mathbf{V}_{4}, \mathbf{V}_{3} \vee \mathbf{V}_{5}, \mathbf{V}_{4} \vee \mathbf{V}_{5}, \mathbf{V}_{1} \vee \mathbf{V}_{4} \vee \mathbf{V}_{5}, \mathbf{V}_{3} \vee \mathbf{V}_{4} \vee \mathbf{V}_{5}=\mathbf{L}$ 
For the sake of conciseness in the analysis that follows including Theorem 5.8, and unless otherwise stated, whenever we consider $L_{6}(m, n)$ or $\mathbf{V}_{m, n}$ it is implicit that $3 \leq n<\omega$ and $0 \leq m \leq n$.

Now observe that

(1) $\mathbf{V}_{4} \subseteq \mathbf{V}_{m, n}$ if and only if $m \geq 1$,

(2) $\mathbf{V}_{5} \subseteq \mathbf{V}_{m, n}$ if and only if $(m, n) \neq(3,3)$.

Let $\mathbf{V}$ be a subvariety of $\mathbf{M}_{1}$ such that $L_{1} \notin \mathbf{V}$ and $L_{6}(m, n) \in \mathbf{V}$, for some $m, n$. Then $L_{3} \notin \mathbf{V}$. Consider

$$
\begin{gathered}
S=\left\{n: L_{6}(n, n) \in \mathbf{V}\right\} \text { and } \\
T=\left\{m: L_{6}(m, n) \in \mathbf{V}, \text { for some } n>m\right\} .
\end{gathered}
$$

We have that $S \neq \emptyset$ or $T \neq \emptyset$. We analyse the various possibilities for $S$ and $T$.

(1) $S=\emptyset$. Then $T \neq \emptyset$.

(1.1) $T$ is infinite. Consider $L_{6}(p, q)$ with $p<q$. As $T$ is infinite, there exists $m \in T$ such that $m \geq 2 q-p>p$. Since $m \in T$, we have that $L_{6}(m, n) \in \mathbf{V}$ for some $n>m$ and $L_{6}(p, q) \in \mathbf{V}_{m, n} \subseteq \mathbf{V}$, by Theorem [5.6. So $\mathbf{V}_{\omega, \omega} \subseteq \mathbf{V}$. Since $L_{0}, L_{2}, L_{4}, L_{5} \in \mathbf{V}_{\omega, \omega}$ and $S=\emptyset$, we conclude that $\mathbf{V}=\mathbf{V}_{\omega, \omega}$.

(1.2) $T$ is finite. Let $T=\left\{p_{0}, \ldots, p_{s}\right\}$ with $s<\omega$ and $p_{0}>p_{1}>\cdots>p_{s}$. For each $i \in\{0, \ldots, s\}$, let $A_{i}=\left\{n: n>p_{i}\right.$ and $\left.L_{6}\left(p_{i}, n\right) \in \mathbf{V}\right\}$ which is a non-empty set.

(1.2.1) $A_{i}$ is finite, for all $i \in\{0, \ldots, s\}$. Let $q_{i}=\max A_{i}$. By Theorem 5.6. for any $i \in$ $\{0, \ldots, s\}$ and any $n \geq 3$ such that $p_{i}<n \leq q_{i}$, we have $L_{6}\left(p_{i}, n\right) \in \mathbf{V}_{p_{i}, q_{i}} \subseteq \mathbf{V}$. If $p_{0}=0$, then $T=\{0\}$ and, $\mathbf{V}=\mathbf{V}_{0, q_{0}}$ or $\mathbf{V}=\mathbf{V}_{0, q_{0}} \vee \mathbf{V}_{4}$, since $L_{0}, L_{2}, L_{5} \in \mathbf{V}_{0, q_{0}}$ but $L_{4} \notin \mathbf{V}_{0, q_{0}}$. Let $p_{0} \geq 1$. As $1 \leq p_{0}<q_{0}$, we have that $L_{0}, L_{2}, L_{4}, L_{5} \in \mathbf{V}_{p_{0}, q_{0}}$ and then $\mathbf{V}=\mathbf{V}_{p_{0}, q_{0}} \vee \cdots \vee \mathbf{V}_{p_{s}, q_{s}}$.

(1.2.2) There exists $i, 0 \leq i \leq s$, such that $A_{i}$ is infinite. Let $M$ be the least such $i$. For any $q>p_{M}, 3 \leq q<\omega$, there exists $n \in A_{M}$ such that $n \geq q$. So $L_{6}\left(p_{M}, n\right) \in \mathbf{V}$ and, by Theorem 5.6. $L_{6}\left(p_{M}, q\right) \in \mathbf{V}_{p_{M}, n} \subseteq \mathbf{V}$. Thus, $\mathbf{V}_{p_{M}, \omega} \subseteq \mathbf{V}$. Moreover, for $0 \leq p \leq p_{M}$ and $q>p, 3 \leq q<\omega$, taking $n$ such that $n>\max \left\{2, p_{M}, q+p_{M}-p\right\}$, we have that $L_{6}(p, q) \in \mathbf{V}_{p_{M}, n} \subseteq \mathbf{V}_{p_{M}, \omega} \subseteq \mathbf{V}$. If $p_{0}=0$, then $T=\{0\}$ and $\mathbf{V}=\mathbf{V}_{0, \omega}$ or $\mathbf{V}=\mathbf{V}_{0, \omega} \vee \mathbf{V}_{4}$, since $L_{0}, L_{2}, L_{5} \in \mathbf{V}_{0, \omega}$ and $L_{4} \notin \mathbf{V}_{0, \omega}$. Suppose $p_{0} \geq 1$. If $M=0$, then $\mathbf{V}=\mathbf{V}_{p_{0}, \omega}$, since $L_{0}, L_{2}, L_{4}, L_{5} \in \mathbf{V}_{p_{0}, \omega}$. If $M \geq 1$, as $A_{0}, \ldots, A_{M-1}$ are finite, arguing as in (1.2.1), we get $\mathbf{V}=\mathbf{V}_{p_{0}, q_{0}} \vee \cdots \vee \mathbf{V}_{p_{M-1}, q_{M-1}} \vee \mathbf{V}_{p_{M}, \omega}$.

(2) $S \neq \emptyset$.

(2.1) $S$ is infinite. Consider $L_{6}(p, q)$ with $p<q$. As $S$ is infinite, there exists $n \in S$ such that $n \geq 2 q-p$. Then $p<n$. As $n \in S, L_{6}(n, n) \in \mathbf{V}$. Since $p<n, p<q$ and $p+\lfloor(n-p) / 2\rfloor+n-n \geq$ $p+(q-p)=q$, we have that $L_{6}(p, q) \in \mathbf{V}_{n, n}$, by Theorem [5.6. So $\mathbf{V}_{\omega, \omega} \subseteq \bigvee_{n \in S} \mathbf{V}_{n, n}$. As $L_{0}, L_{2}, L_{4}, L_{5} \in \mathbf{V}_{\omega, \omega}$, we conclude that $\mathbf{V}=\bigvee_{n \in S} \mathbf{V}_{n, n}$.

(2.2) $S$ is finite. Let $S=\left\{n_{0}, \ldots, n_{k}\right\}$ with $k<\omega$ and $n_{0}>n_{1}>\cdots>n_{k}$.

(2.2.1) $T=\emptyset$. Then, for any $m, n$ such that $L_{6}(m, n) \in \mathbf{V}$, we have $m=n$. If $n_{0} \geq 4$, then $L_{6}(2,3) \in \mathbf{V}_{n_{0}, n_{0}} \subseteq \mathbf{V}$, a contradiction. So $n_{0}=3$ and $S=\{3\}$. As $L_{0}, L_{2}, L_{4} \in \mathbf{V}_{3,3}$ but $L_{5} \notin \mathbf{V}_{3,3}$, we conclude that $\mathbf{V}=\mathbf{V}_{3,3}$ or $\mathbf{V}=\mathbf{V}_{3,3} \vee \mathbf{V}_{5}$.

(2.2.2) $T \neq \emptyset$. We are going to apply what was done in (1). Notice that $L_{4} \in \mathbf{V}_{n, n}$, for any $3 \leq n<\omega$. If $T$ is infinite, then $\mathbf{V}=\mathbf{V}_{\omega, \omega} \vee \mathbf{V}_{n_{0}, n_{0}} \vee \cdots \vee \mathbf{V}_{n_{k}, n_{k}}$. If $T=\left\{p_{0}, \ldots, p_{s}\right\}$ with $s<\omega$ and $p_{0}>p_{1}>\cdots>p_{s}$, then $\mathbf{V}=\mathbf{V}_{p_{0}, q_{0}} \vee \cdots \vee \mathbf{V}_{p_{s}, q_{s}} \vee \mathbf{V}_{n_{0}, n_{0}} \vee \cdots \vee \mathbf{V}_{n_{k}, n_{k}}$, or $\mathbf{V}=$ $\mathbf{V}_{p, \omega} \vee \mathbf{V}_{n_{0}, n_{0}} \vee \cdots \vee \mathbf{V}_{n_{k}, n_{k}}$ for some $p<\omega$, or $\mathbf{V}=\mathbf{V}_{p_{0}, q_{0}} \vee \cdots \vee \mathbf{V}_{p_{t}, q_{t}} \vee \mathbf{V}_{p, \omega} \vee \mathbf{V}_{n_{0}, n_{0}} \vee \cdots \vee \mathbf{V}_{n_{k}, n_{k}}$ for some $t<\omega$ and $p_{0}>\cdots>p_{t}>p$.

Losing some of the detail from above, the structure of $L_{V}\left(\mathbf{M}_{1}\right)$ may be summarised as follows. 
Let $\left(\mathcal{K}_{i}: i<2^{\omega}\right)$ be the family of infinite subsets of $\left\{L_{6}(n, n): 3 \leq n<\omega\right\}$.

THEOREM 5.8. Every non-trivial subvariety $\mathbf{V}$ of $\mathbf{M}_{1}$ may be expressed as follows

(1) $\mathbf{V}_{i}, 0 \leq i \leq 5, \mathbf{V}_{1} \vee \mathbf{V}_{2}, \mathbf{V}_{1} \vee \mathbf{V}_{4}, \mathbf{V}_{1} \vee \mathbf{V}_{5}, \mathbf{V}_{3} \vee \mathbf{V}_{4}, \mathbf{V}_{3} \vee \mathbf{V}_{5}, \mathbf{V}_{4} \vee \mathbf{V}_{5}, \mathbf{V}_{1} \vee \mathbf{V}_{4} \vee \mathbf{V}_{5}$, $\mathbf{V}_{3} \vee \mathbf{V}_{4} \vee \mathbf{V}_{5}$

(2) $\mathbf{V}_{3,3} \vee \mathbf{V}_{5}, \mathbf{V}_{0, \omega} \vee \mathbf{V}_{4}$ and $\mathbf{V}_{0, q} \vee \mathbf{V}_{4}$ for some $3 \leq q<\omega$.

(3) $\mathbf{V}_{p_{0}, q_{0}} \vee \cdots \vee \mathbf{V}_{p_{s-1}, q_{s-1}} \vee \mathbf{V}_{n_{0}, n_{0}} \vee \cdots \vee \mathbf{V}_{n_{k-1}, n_{k-1}}$, for some $s, k<\omega$ such that $s+k>0$ where $p_{i}<q_{i}$ for any $0 \leq i<s, p_{0}>\cdots>p_{s-1}$ and $n_{0}>\cdots>n_{k-1}$.

(4) $\mathbf{V}_{p_{0}, q_{0}} \vee \cdots \vee \mathbf{V}_{p_{s-1}, q_{s-1}} \vee \mathbf{V}_{p, \omega} \vee \mathbf{V}_{n_{0}, n_{0}} \vee \cdots \vee \mathbf{V}_{n_{k-1}, n_{k-1}}$, for some $s, k<\omega, p<\omega$ where $p<p_{i}<q_{i}$, for any $0 \leq i<s, p_{0}>\cdots>p_{s-1}>p$ and $n_{0}>\cdots>n_{k-1}$.

(5) $\mathbf{V}_{\omega, \omega} \vee \mathbf{V}_{n_{0}, n_{0}} \vee \cdots \vee \mathbf{V}_{n_{k-1}, n_{k-1}}$ for some $k<\omega$ and $n_{0}>\cdots>n_{k-1}$.

(6) $\mathbf{V}\left(\mathcal{K}_{i}\right)$ for $i<2^{\omega}$.

(7) $\mathbf{V} \vee \mathbf{V}_{1}$ and $\mathbf{V} \vee \mathbf{V}_{3}$ where $\mathbf{V}$ is one of the ones described in (2)-(6).

Observe that $\mathbf{M}_{\mathbf{1}}=\mathbf{V}_{3} \vee \mathbf{V}\left(\left\{L_{6}(n, n): 3 \leq n<\omega\right\}\right)$.

Also notice that the representation of the subvarieties described in (3), (4) and, consequently, (7) might be redundant. For example, $\mathbf{V}_{7,8} \vee \mathbf{V}_{3,6}=\mathbf{V}_{7,8}$ and, although $\mathbf{V}_{7,8} \vee \mathbf{V}_{2,6}$ is an irredundant representation, we have that $\mathbf{V}_{7,8} \vee \mathbf{V}_{2,6} \vee \mathbf{V}_{9,9}=\mathbf{V}_{2,6} \vee \mathbf{V}_{9,9}$.

It is possible to obtain irredundant representations for all of the aforementioned subvarieties, but that requires some technical arguments and it does not add much to the understanding of the lattice $L_{V}\left(\mathbf{M}_{1}\right)$. For example, in (3) we obtain $\mathbf{V}_{p_{0}, q_{0}} \vee \cdots \mathbf{V}_{p_{s-1}, q_{s-1}} \vee \mathbf{V}_{p_{s}, q_{s}} \vee \cdots \vee$ $\mathbf{V}_{p_{s+t-1}, q_{s+t-1}} \vee \mathbf{V}_{n_{0}, n_{0}} \vee \cdots \vee \mathbf{V}_{n_{k-1}, n_{k-1}}$, for some $s, t, k<\omega$ such that $s+t+k>0$ where $p_{0}>\cdots>p_{s-1}>n_{0} \geq p_{s}>\cdots>p_{s+t-1}, n_{0}>\cdots>n_{k-1}$, and, for any $0 \leq i<s+t, p_{i}<q_{i}$, $q_{i}>p_{i}+\left\lfloor\left(p_{i-1}-p_{i}\right) / 2\right\rfloor+q_{i-1}-p_{i-1}$ and $q_{s}>p_{s}+\left\lfloor\left(n_{0}-p_{s}\right) / 2\right\rfloor$.

\section{The lattice of subvarieties of $\mathbf{K}_{2}$}

In this section we show that $L_{V}\left(\mathbf{K}_{\mathbf{2}}\right)$ is uncountable. Recall that we already know that $\mathbf{K}_{\mathbf{2}}$ is not locally finite, as seen in Theorem 4.13, and that $L_{V}\left(\mathbf{K}_{\mathbf{1}}\right)$ is an $\omega+1$ chain, as seen in Theorem 5.4 Toward this end, the following sequence of regular $p m$-algebras plays a crucial role.

EXAMPLE 6.1. For $2 \leq n<\omega$, let $A_{n}=\left\{a_{i}: 0 \leq i<n\right\}$ and $B_{n}=\left\{b_{i}: 0 \leq i<n\right\}$ be disjoint $n$-element sets. Consider $S_{n}=A_{n} \cup B_{n}$ and a disjoint copy $\zeta\left(S_{n}\right)$. Extend $\zeta$ by defining $\zeta(\zeta(x))=x$, for every $x \in S_{n}$. Let $\leq$ be the partial order induced by, for $0 \leq i, j<n$,

$$
\begin{gathered}
a_{i}<\zeta\left(a_{j}\right), b_{i}<\zeta\left(b_{j}\right), \\
a_{i}<\zeta\left(b_{j}\right) \text { iff } i \neq j, \text { and } b_{i}<\zeta\left(a_{j}\right) \text { iff } i \neq j .
\end{gathered}
$$

It is straightforward to prove that $P_{n}=\left(S_{n} \cup \zeta\left(S_{n}\right) ; \leq, \zeta\right)$ is a $p m$-space. Let $K_{n}=E\left(P_{n}\right)$. Observe that, since $a_{i}<\zeta\left(a_{i}\right)$ and $b_{i}<\zeta\left(b_{i}\right)$ for every $0 \leq i<n, K_{n}$ is a pseudocomplemented Kleene algebra and, as $P_{n}$ has height $1, K_{n}$ is a regular pseudocomplemented Kleene algebra.

LEMMA 6.2. (1) For $2 \leq n<\omega$, the regular pseudocomplemented Kleene algebra $K_{n}$ is a simple algebra of $\mathbf{K}_{2}$.

(2) For $2 \leq n, m<\omega$, if $K_{n} \in \mathbf{I S}\left(K_{m}\right)$, then $n=m$. 
Proof. (1) Applying Corollary 4.3, it is sufficient to prove that, for any $x, y \in P_{n}$, we have $\ell(x, y) \leq 2$ or $\ell(x, \zeta(y)) \leq 2$. Suppose that, for distinct $i$ and $j, 0 \leq i, j<n, x \in\left\{a_{i}, b_{i}\right\}$ and $y \in\left\{a_{j}, b_{j}\right\}$. Then $x<\zeta(x)$ and $\zeta(x)>y$. So $\ell(x, y)=2$. For $0 \leq i<n$, we have $\ell\left(a_{i}, b_{i}\right)=2$, since, choosing $j \neq i, a_{i}<\zeta\left(b_{j}\right)$ and $\zeta\left(b_{j}\right)>b_{i}$. So, for any $x, y \in S_{n}, \ell(x, y) \leq 2$ and, consequently, $\ell(\zeta(x), \zeta(y)) \leq 2$. From this fact we also conclude that if either $x \in S_{n}$ and $y \in \zeta\left(S_{n}\right)$, or $x \in \zeta\left(S_{n}\right)$ and $y \in S_{n}$, then $\ell(x, \zeta(y)) \leq 2$.

(2) Suppose $K_{n} \in \mathbf{I S}\left(K_{m}\right)$. Then there exists a surjective pm-morphism $\varphi: P_{m} \rightarrow P_{n}$. It is obvious that $m \geq n$. Suppose $m>n$. For such a $\varphi$, we know that $\varphi\left(S_{m}\right)=\varphi\left(\operatorname{Min}\left(P_{m}\right)\right)=$ $\operatorname{Min}\left(P_{n}\right)=S_{n}$ and $\varphi\left(\zeta\left(S_{m}\right)\right)=\zeta\left(\varphi\left(S_{m}\right)\right)=\zeta\left(S_{n}\right)$. As $\left|S_{m}\right|=2 m,\left|S_{n}\right|=2 n$ and $2 m>2 n$, there exist $x, y \in S_{m}$ such that $x \neq y$ and $\varphi(x)=\varphi(y)$. Since $x, y$ are distinct elements of $S_{m}$, we have that, for any $z \in S_{m}, z<\zeta(x)$ or $z<\zeta(y)$, and then $\varphi(z)<\varphi(\zeta(x))=\zeta(\varphi(x))$ or $\varphi(z)<\varphi(\zeta(y))=\zeta(\varphi(y))$. But $\varphi(x)=\varphi(y)$, so, for any $z \in S_{m}, \varphi(z)<\zeta(\varphi(x))$. If $\varphi(x)=a_{i}$, for some $0 \leq i<n$, then $b_{i} \notin \varphi\left(S_{m}\right)$, since $b_{i} \nless \zeta\left(a_{i}\right)$. If $\varphi(x)=b_{i}$, for some $0 \leq i<n$, then $a_{i} \notin \varphi\left(S_{m}\right)$, since $a_{i} \nless \zeta\left(b_{i}\right)$. Either way, we have reached a contradiction. Thus, $m=n$.

Notice that, for any $2 \leq n<\omega, K_{n} \notin \mathbf{K}_{1}$, since $\ell_{\zeta}\left(a_{0}, b_{0}\right)=2$, as $\ell\left(a_{0}, b_{0}\right)=2$ and $\ell\left(a_{0}, \zeta\left(b_{0}\right)\right)=3$.

Consider $\mathbf{V}\left(\left\{K_{n}: 2 \leq n<\omega\right\}\right)$ which is a subvariety of $\mathbf{K}_{2}$. Although, as shown in Theorem $4.13 \mathbf{K}_{2}$ is not locally finite, as we will now prove, $\mathbf{V}\left(\left\{K_{n}: 2 \leq n<\omega\right\}\right)$ is, our aim being to establish Theorem 6.5.

LEMMA 6.3. The variety $\mathbf{V}\left(\left\{K_{n}: 2 \leq n<\omega\right\}\right)$ is locally finite.

Proof. As in Theorem 4.10, it is sufficient to show that for each $N<\omega$, there exists $N^{\prime}<\omega$, depending on $N$, such that any set of $N$ elements in any one of the pseudocomplemented Kleene algebras $K_{n}, 2 \leq n<\omega$, generates a subalgebra that has at most $N^{\prime}$ elements.

Let $2 \leq n<\omega$ and consider the $p m$-space $P_{n}$. Observe that, for each $i, 0 \leq i<n$, we have

$$
\left(\zeta\left(a_{i}\right)\right]=\left\{\zeta\left(a_{i}\right)\right\} \cup\left(S_{n} \backslash\left\{b_{i}\right\}\right) \text { and }\left(\zeta\left(b_{i}\right)\right]=\left\{\zeta\left(b_{i}\right)\right\} \cup\left(S_{n} \backslash\left\{a_{i}\right\}\right) .
$$

A claim analogous to the one in Theorem 4.10 holds in this context too.

For any set $C$, let $\mathcal{P}(C)$ denote the Boolean algebra of all subsets of $C$.

Claim. Let $\mathcal{F}$ be a Boolean subalgebra of the Boolean algebra $\mathcal{P}\left(S_{n}\right)$ such that, for $0 \leq i<n$, $\left\{a_{i}\right\} \in \mathcal{F}$ if and only if $\left\{b_{i}\right\} \in \mathcal{F}$, let $\mathcal{F}_{I}$ be the set of singletons of $\mathcal{F}$ and

$$
K(\mathcal{F}):=\mathcal{F} \cup\left\{\zeta(X) \cup S_{n}: X \in \mathcal{F}\right\} \cup\left\{(\zeta(x)]:\{x\} \in \mathcal{F}_{I}\right\} .
$$

Then $K(\mathcal{F})$ is a subalgebra of $K_{n}$.

The elements of $K(\mathcal{F})$ are decreasing sets of $P_{n}$.

As in the proof of the claim in Theorem 4.10 $\emptyset \in K(\mathcal{F}), S_{n} \cup \zeta\left(S_{n}\right) \in K(\mathcal{F})$ and finite unions and intersections of elements in $\mathcal{F} \cup\left\{\zeta(X) \cup S_{n}: X \in \mathcal{F}\right\}$ belong to $K(\mathcal{F})$. Now, let $X \in \mathcal{F}$ and $\{x\} \in \mathcal{F}_{I}$. Suppose $x=a_{i}$, for some $0 \leq i<n$ (for $x=b_{i}$ it is analogous). Then $\left\{a_{i}\right\} \in \mathcal{F}$ and, consequently, $\left\{b_{i}\right\} \in \mathcal{F}$. If $b_{i} \in X$, then $X \cup\left(\zeta\left(a_{i}\right)\right]=X \cup\left\{\zeta\left(a_{i}\right)\right\} \cup\left(S_{n} \backslash\left\{b_{i}\right\}\right)=\zeta\left(\left\{a_{i}\right\}\right) \cup S_{n} \in K(\mathcal{F})$, whilst, if $b_{i} \notin X, X \cup\left(\zeta\left(a_{i}\right)\right]=\left(\zeta\left(a_{i}\right)\right]$. Moreover, $X \cap\left(\zeta\left(a_{i}\right)\right]=X \cap\left(S_{n} \backslash\left\{b_{i}\right\}\right) \in \mathcal{F} \subseteq K(\mathcal{F})$. Further, we 
have $\left(\zeta(X) \cup S_{n}\right) \cup\left(\zeta\left(a_{i}\right)\right]=\zeta\left(X \cup\left\{a_{i}\right\}\right) \cup S_{n} \in K(\mathcal{F})$ and, if $a_{i} \in X,\left(\zeta(X) \cup S_{n}\right) \cap\left(\zeta\left(a_{i}\right)\right]=\left(\zeta\left(a_{i}\right)\right]$, otherwise $\left(\zeta(X) \cup S_{n}\right) \cap\left(\zeta\left(a_{i}\right)\right]=S_{n} \backslash\left\{b_{i}\right\} \in \mathcal{F} \subseteq K(\mathcal{F})$. Finally, let $\{y\} \in \mathcal{F}_{I}$ such that $y \neq a_{i}$. Then $\{y\} \in \mathcal{F}$ and $\left\{a_{i}, y\right\} \in \mathcal{F}$ and we have $\left(\zeta\left(a_{i}\right)\right] \cup(\zeta(y)]=\zeta\left(\left\{a_{i}, y\right\}\right) \cup S_{n} \in K(\mathcal{F})$. If $y=a_{j}$ with $j \neq i$, then $\left\{b_{j}\right\} \in \mathcal{F}$ and $\left(\zeta\left(a_{i}\right)\right] \cap(\zeta(y)]=\left(\zeta\left(a_{i}\right)\right] \cap\left(\zeta\left(a_{j}\right)\right]=S_{n} \backslash\left\{b_{i}, b_{j}\right\} \in \mathcal{F} \subseteq K(\mathcal{F})$. If $y=b_{j}, 0 \leq j<n$, then $\left\{a_{j}\right\} \in \mathcal{F}$ and $\left(\zeta\left(a_{i}\right)\right] \cap(\zeta(y)]=\left(\zeta\left(a_{i}\right)\right] \cap\left(\zeta\left(b_{j}\right)\right]=S_{n} \backslash\left\{b_{i}, a_{j}\right\} \in \mathcal{F} \subseteq K(\mathcal{F})$. Thus $K(\mathcal{F})$ is closed under unions and intersections.

Now we show that $K(\mathcal{F})$ is closed under pseudocomplementation. Let $X \in \mathcal{F}$. We have $X^{*}=\left(S_{n} \cup \zeta\left(S_{n}\right)\right) \backslash[X)=S_{n} \backslash X$, unless $X=\emptyset$ or $|X|=1$. In the first case, $X^{*}=S_{n} \cup \zeta\left(S_{n}\right)$. In the latter, we have $X=\left\{a_{i}\right\}$ or $X=\left\{b_{i}\right\}$, for some $0 \leq i<n$, and then both $\left\{a_{i}\right\}$ and $\left\{b_{i}\right\}$ belong to $\mathcal{F}_{I}$ and $\left\{a_{i}\right\}^{*}=\left(\zeta\left(b_{i}\right)\right] \in K(\mathcal{F}),\left\{b_{i}\right\}^{*}=\left(\zeta\left(a_{i}\right)\right] \in K(\mathcal{F})$. For $\zeta(X) \cup S_{n}$, we have $\left(\zeta(X) \cup S_{n}\right)^{*}=\emptyset$. Finally, if $\left\{a_{i}\right\} \in \mathcal{F}_{I}$, for some $0 \leq i<n$, then $\left\{a_{i}\right\} \in \mathcal{F}$ and $\left\{b_{i}\right\} \in \mathcal{F}$, and we have $\left(\zeta\left(a_{i}\right)\right]^{*}=\left\{b_{i}\right\} \in \mathcal{F} \subseteq K(\mathcal{F})$. Analogously, $\left(\zeta\left(b_{i}\right)\right]^{*}=\left\{a_{i}\right\} \in \mathcal{F} \subseteq K(\mathcal{F})$, if $\left\{b_{i}\right\} \in \mathcal{F}_{I}$, for some $0 \leq i<n$.

It remains to show that $K(\mathcal{F})$ is closed for the de Morgan unary operation. Let $X \in \mathcal{F}$. We have $X^{\prime}=\left(S_{n} \cup \zeta\left(S_{n}\right)\right) \backslash \zeta(X)=\zeta\left(S_{n} \backslash X\right) \cup S_{n} \in K(\mathcal{F})$ and $\left(\zeta(X) \cup S_{n}\right)^{\prime}=\left(S_{n} \cup \zeta\left(S_{n}\right)\right) \backslash \zeta\left(\zeta(X) \cup S_{n}\right)=$ $S_{n} \backslash X \in \mathcal{F} \subseteq K(\mathcal{F})$. For the third type of elements in $K(\mathcal{F})$, we have $\left\{a_{i}\right\} \in \mathcal{F}_{I}$ if and only if $\left\{b_{i}\right\} \in \mathcal{F}_{I}$, and $\left(\zeta\left(a_{i}\right)\right]^{\prime}=\left(\zeta\left(b_{i}\right)\right] \in K(\mathcal{F})$ and $\left(\zeta\left(b_{i}\right)\right]^{\prime}=\left(\zeta\left(a_{i}\right)\right] \in K(\mathcal{F})$.

The proof of the claim is now complete.

Let $N$ be a positive integer. Let $\left\{X_{i}: 0 \leq i<N\right\}$ be a set of decreasing sets of $P_{n}$. For each $i, 0 \leq i<N$, we have that $X_{i}=\left(X_{i} \cap S_{n}\right) \cup\left(X_{i} \cap \zeta\left(S_{n}\right)\right)=\left(X_{i} \cap S_{n}\right) \cup \zeta\left(\zeta\left(X_{i}\right) \cap S_{n}\right)$ and $X_{i} \cap \bar{S}_{n}=\left(X_{i} \cap A_{n}\right) \cup\left(X_{i} \cap B_{n}\right)$ and $\zeta\left(X_{i}\right) \cap S_{n}=\left(\zeta\left(X_{i}\right) \cap A_{n}\right) \cup\left(\zeta\left(X_{i}\right) \cap B_{n}\right)$. Consider $T=\left\{X_{i}: 0 \leq i<N\right\} \cup\left\{\zeta\left(X_{i}\right): 0 \leq i<N\right\}$. This set has, at most, $N_{1}=2 N$ elements.

For each $Y \subseteq A_{n}$ and each $Z \subseteq B_{n}$, let $B(Y)=\left\{b_{i}: a_{i} \in Y\right\}$ and $A(Z)=\left\{a_{i}: b_{i} \in Z\right\}$. Clearly, for any $Y \subseteq A_{n}$ and $Z \subseteq B_{n}$ we have $A(B(Y))=Y, B(A(Z))=Z, B\left(A_{n} \backslash Y\right)=B_{n} \backslash B(Y)$ and $A\left(B_{n} \backslash Z\right)=A_{n} \backslash A(Z)$. It is also clear that $B\left(Y_{1} \cap Y_{2}\right)=B\left(Y_{1}\right) \cap B\left(Y_{2}\right), A\left(Z_{1} \cap Z_{2}\right)=A\left(Z_{1}\right) \cap A\left(Z_{2}\right)$, for any $Y_{1}, Y_{2} \subseteq A_{n}$ and $Z_{1}, Z_{2} \subseteq B_{n}$. Consider

$\mathcal{A}=\left\{X \cap A_{n}: X \in T\right\} \cup\left\{A\left(X \cap B_{n}\right): X \in T\right\}$ and $\mathcal{B}=\left\{X \cap B_{n}: X \in T\right\} \cup\left\{B\left(X \cap A_{n}\right): X \in T\right\}$.

Obviously, each of the sets $\mathcal{A}$ and $\mathcal{B}$ has, at most, $N_{2}=2 N_{1}$ elements. Let $\mathcal{F}_{\mathcal{A}}$ be the Boolean subalgebra of the Boolean algebra $\mathcal{P}\left(A_{n}\right)$ generated by $\mathcal{A}$ and $\mathcal{F}_{\mathcal{B}}$ be the Boolean subalgebra of the Boolean algebra $\mathcal{P}\left(B_{n}\right)$ generated by $\mathcal{B}$ and let $\mathcal{F}=\left\{Y \cup Z: Y \in \mathcal{F}_{\mathcal{A}}\right.$ and $\left.Z \in \mathcal{F}_{\mathcal{B}}\right\}$ which is a Boolean subalgebra of $\mathcal{P}\left(S_{n}\right)$, since $S_{n}$ is the disjoint union of $A_{n}$ and $B_{n}$. Since a free Boolean algebra on $N_{2}$ generators has $2^{\left(2^{N_{2}}\right)}$ elements, each one of $\mathcal{F}_{\mathcal{A}}$ and $\mathcal{F}_{\mathcal{B}}$ has, at most, $N_{3}=2^{\left(2^{N_{2}}\right)}$ elements, and so, the cardinality of $\mathcal{F}$ is less than or equal to $N_{4}=\left(N_{3}\right)^{2}$. Now, suppose that $\left\{a_{i}\right\} \in \mathcal{F}, 0 \leq i<n$. Then $\left\{a_{i}\right\} \in \mathcal{F}_{\mathcal{A}}$ and, since $\mathcal{F}_{\mathcal{A}}$ is generated by $\mathcal{A}$ and $\left\{a_{i}\right\}$ is an atom, we have $\left\{a_{i}\right\}=W_{1} \cap \cdots \cap W_{k}$, where $1 \leq k<\omega$, and, for each $j, 1 \leq j \leq k, W_{j} \in \mathcal{A}$ or $A_{n} \backslash W_{j} \in \mathcal{A}$. So $\left\{b_{i}\right\}=B\left(\left\{a_{i}\right\}\right)=B\left(W_{1}\right) \cap \cdots \cap B\left(W_{k}\right)$ and, for each $j, 1 \leq j \leq k, B\left(W_{j}\right) \in \mathcal{B}$ or $B_{n} \backslash B\left(W_{j}\right)=B\left(A_{n} \backslash W_{j}\right) \in \mathcal{B}$. Thus $\left\{b_{i}\right\} \in \mathcal{F}_{\mathcal{B}} \subseteq \mathcal{F}$. Analogously, we prove that if $\left\{b_{i}\right\} \in \mathcal{F}$, $0 \leq i<n$, then $\left\{a_{i}\right\} \in \mathcal{F}$.

By the above claim, $K(\mathcal{F})$ is a subalgebra of $K_{n}$. This subalgebra has, at most, $3 N_{4}$ elements. It remains to show that, for any $i, 0 \leq i<N, X_{i} \in K(\mathcal{F})$. We have that $X_{i}=\left(X_{i} \cap S_{n}\right) \cup \zeta\left(\zeta\left(X_{i}\right) \cap S_{n}\right)$ and $X_{i} \cap S_{n}=\left(X_{i} \cap A_{n}\right) \cup\left(X_{i} \cap B_{n}\right)$ and $\zeta\left(X_{i}\right) \cap S_{n}=\left(\zeta\left(X_{i}\right) \cap A_{n}\right) \cup\left(\zeta\left(X_{i}\right) \cap B_{n}\right)$. If $\zeta\left(X_{i}\right) \cap S_{n}=\emptyset$, then $X_{i}=\left(X_{i} \cap A_{n}\right) \cup\left(X_{i} \cap B_{n}\right) \in \mathcal{F} \subseteq K(\mathcal{F})$ since, as $X_{i} \in T, X_{i} \cap A_{n} \in \mathcal{A} \subseteq \mathcal{F}_{\mathcal{A}}$ and 
$X_{i} \cap B_{n} \in \mathcal{B} \subseteq \mathcal{F}_{\mathcal{B}}$. Now, suppose $\zeta\left(X_{i}\right) \cap S_{n}=\left\{a_{j}\right\}$ for some $0 \leq j<n$. Then $\zeta\left(X_{i}\right) \cap A_{n}=\left\{a_{j}\right\}$. As $\zeta\left(X_{i}\right) \in \bar{T}$, we have $\left\{a_{j}\right\} \in \mathcal{A} \subseteq \mathcal{F}_{\mathcal{A}} \subseteq \mathcal{F}$. As $\left\{a_{j}\right\}$ is a singleton, it belongs to $\mathcal{F}_{I}$. Since $\zeta\left(X_{i}\right) \cap S_{n}=\left\{a_{j}\right\}$, we have $\zeta\left(\zeta\left(X_{i}\right) \cap S_{n}\right)=\left\{\zeta\left(a_{j}\right)\right\}$ and $X_{i}=\left(X_{i} \cap S_{n}\right) \cup\left\{\zeta\left(a_{j}\right)\right\}$. But $X_{i}$ is decreasing, so either $X_{i}=\left\{\zeta\left(a_{j}\right)\right\} \cup\left(S_{n} \backslash\left\{b_{j}\right\}\right)=\left(\zeta\left(a_{j}\right)\right]$ or $X_{i}=\zeta\left(\left\{a_{j}\right\}\right) \cup S_{n}$. Either way, $X_{i} \in K(\mathcal{F})$. We prove similarly that if $\zeta\left(X_{i}\right) \cap S_{n}=\left\{b_{j}\right\}$ for some $0 \leq j<n$, then $X_{i} \in K(\mathcal{F})$. Finally, suppose $\left|\zeta\left(X_{i}\right) \cap S_{n}\right| \geq 2$. As $X_{i} \cap \zeta\left(S_{n}\right)=\zeta\left(\zeta\left(X_{i}\right) \cap S_{n}\right)$, we have $\left|X_{i} \cap \zeta\left(S_{n}\right)\right| \geq 2$ and, since $X_{i}$ is decreasing, $S_{n} \subseteq X_{i}$. So $X_{i}=\zeta\left(\zeta\left(X_{i}\right) \cap S_{n}\right) \cup S_{n}$. But $\zeta\left(X_{i}\right) \cap S_{n}=\left(\zeta\left(X_{i}\right) \cap A_{n}\right) \cup\left(\zeta\left(X_{i}\right) \cap B_{n}\right)$ and, as $\zeta\left(X_{i}\right) \in T$, we have $\zeta\left(X_{i}\right) \cap A_{n} \in \mathcal{A} \subseteq \mathcal{F}_{\mathcal{A}}$ and $\zeta\left(X_{i}\right) \cap B_{n} \in \mathcal{B} \subseteq \mathcal{F}_{\mathcal{B}}$. Consequently, $\zeta\left(X_{i}\right) \cap S_{n} \in \mathcal{F}$ and $X_{i} \in K(\mathcal{F})$.

LEMMA 6.4. $\left|L_{V}\left(\mathbf{V}\left(\left\{K_{n}: 2 \leq n<\omega\right\}\right)\right)\right|=2^{\omega}$.

Proof. Let $\mathcal{K}$ and $\mathcal{K}^{\prime}$ be non-empty subsets of $\left\{K_{n}: 2 \leq n<\omega\right\}$ such that $\mathcal{K} \neq \mathcal{K}^{\prime}$. Without loss of generality, we may assume that there exists $K_{n} \in \mathcal{K} \backslash \mathcal{K}^{\prime}$. Obviously, $K_{n} \in \mathbf{V}(\mathcal{K})$. If $K_{n} \in \mathbf{V}\left(\mathcal{K}^{\prime}\right)$, then, applying Theorem [5.2, $K_{n} \in \mathbf{I S}\left(K_{m}\right)$ for some $K_{m} \in \mathcal{K}^{\prime}$ and, by (2) of Lemma 6.2, $n=m$ which is a contradiction. Thus, $K_{n} \notin \mathbf{V}\left(\mathcal{K}^{\prime}\right)$ and $\mathbf{V}(\mathcal{K}) \neq \mathbf{V}\left(\mathcal{K}^{\prime}\right)$.

Since $\mathbf{V}\left(\left\{K_{n}: 2 \leq n<\omega\right\}\right)$ is a subvariety of $\mathbf{K}_{2}$, the following is immediate.

THEOREM 6.5. $\left|L_{V}\left(\mathbf{K}_{2}\right)\right|=2^{\omega}$.

The ad hoc nature of Example 6.1 strongly suggests that, unlike $L_{V}\left(\mathbf{M}_{\mathbf{1}}\right)$ which is also uncountable, the structure of $L_{V}\left(\mathbf{K}_{\mathbf{2}}\right)$ (and so too $L_{V}\left(\mathbf{M}_{\mathbf{2}}\right)$ ) defies a meaningful description.

\section{References}

[1] Adams, M.E., Sankappanavar, H.P., Vaz de Carvalho, J.: Regular double p-algebras. Math. Slovaca 69, 1534 (2019)

[2] R. Balbes and P. Dwinger, Distributive lattices, University of Missouri Press, Columbia, 1974.

[3] Beazer, R.: The determination congruence on double $p$-algebras. Algebra Universalis 6,121-129 (1976)

[4] S. Burris and H. P. Sankappanavar, A Course in Universal Algebra, Springer-Verlag, New York, 1981.

[5] W. H. Cornish and P. R. Fowler, Coproducts of De Morgan algebras, Bull. Austral. Math. Soc. 16 (1977), 1-13.

[6] W. H. Cornish and P. R. Fowler, Coproducts of Kleene algebras, J. Austral. Math. Soc. Ser. A 27 (1979), 209-220.

[7] K. Denecke, Functional completeness in pseudocomplemented De Morgan algebras, Beitr. Algebra Geom. 24 (1987), 135-150.

[8] H. Gaitán, Free algebras in certain varieties of distributive pseudocomplemented De Morgan algebras, Math. Log. Quart. 44 (1998), 553-567.

[9] F. Guzmán and C. Squier, Subdirectly irreducible and free Kleene-Stone algebras, Algebra Universalis 31 (1994), $266-273$.

[10] B. Jónsson, Algebras whose congruence lattices are distributive, Math. Scand. 21 (1967), 110-121.

[11] J. A. Kalman, Lattices with involution, Trans. Amer. Math. Soc. 87 (1958), 485-491.

[12] Koubek, V., Sichler, J.: Categorical universality of regular double p-algebras. Glasgow Math. J. 32, 329340 (1990)

[13] T. Katriňák, The structure of distributive double p-algebras. Regularity and congruences, Algebra Universalis 3 (1973), 238-246.

[14] A. Mal'cev, Algebraicheskie Systemi [in Russian], Nauka Press, Moscow, 1970. English Translation: Algebraic Systems, Academie-Verlag, Berlin, 1973.

[15] R. N. McKenzie, G. F. McNulty and W. F. Taylor, Algebras, Lattices, Varieties, Vol. 1, Wadsworth \& Brooks/Cole, California, 1987. 
[16] H. A. Priestley, Representation of distributive lattices by means of ordered Stone spaces, Bull. London Math. Soc. 2 (1970), 186-190.

[17] H. A. Priestley, The construction of spaces dual to pseudocomplemented distributive lattices, Quart. J. Math. Oxford Ser. (2) 26 (1975), 215-228.

[18] H. A. Priestley, Ordered sets and duality for distributive lattices, Orders: description and roles (L'Arbresle, 1982), 39-60, North-Holland Math. Stud., 99, North-Holland, Amsterdam, 1984.

[19] P. Ribenboim, Characterization of the sup-complement in a distributive lattice with last element, Summa Brasil. Math. 2 (1949). 43-49.

[20] A. Romanowska, Subdirectly irreducible pseudocomplemented De Morgan algebras, Algebra Universalis 12 (1981), 70-75.

[21] H. P. Sankappanavar, Pseudocomplemented Ockham and De Morgan algebras, Z. Math. Logik Grundlag. Math. 32 (1986), 385-394.

[22] H. P. Sankappanavar, Principal congruences of pseudocomplemented De Morgan algebras, Z. Math. Logik Grundlag. Math. 33 (1987), 3-11.

[23] H. P. Sankappanavar, Heyting algebras with a dual lattice endomorphism, Z. Math. Logik Grundlag. Math. 33 (1987), 565-573.

[24] H. P. Sankappanavar and J. Vaz de Carvalho, Congruence properties of pseudocomplemented De Morgan algebras, Math. Log. Quart. 60 (2014), 425-436.

[25] Taylor, C.J.: Algebras of incidence structures: representations of regular double p-algebras. Algebra Universalis 76,479491 (2016)

[26] J. Varlet, Algèbres de Lukasiewicz trivalentes, Bull. Soc. Roy. Sci. Liège 36 (1968), 399-408.

[27] J. Varlet, A regular variety of type $<2,2,1,1,0,0>$, Algebra Universalis 2 (1972), 218-223.

[28] X. Wang and L. Wang, The Lattices of Kernel Ideals in Pseudocomplemented De Morgan Algebras, Order, 34 (2017), 23-35.

Department of Mathematics

State University of New York

New Paltz, NY 12561, USA

adamsm@newpaltz.edu

sankapph@newpaltz.edu
Centro de Matemática e Aplicações Departamento de Matemática

Faculdade de Ciências e Tecnologia Universidade NOVA de Lisboa 2829-516 Caparica, Portugal

jvc@fct.unl.pt 